Research Article

\title{
Fault Diagnosis of Bearings with Adjusted Vibration Spectrum Images
}

\author{
Mingquan Qiu $\mathbb{D}^{1,2}$ Wei Li $\mathbb{D}^{1,2}$ Zhencai Zhu, ${ }^{1,2}$ Fan Jiang $\mathbb{D}^{1,2}$ and Gongbo Zhou $\mathbb{D}^{1,2}$ \\ ${ }^{1}$ School of Mechatronic Engineering, China University of Mining and Technology, Xuzhou 221116, China \\ ${ }^{2}$ Jiangsu Key Laboratory of Mine Mechanical and Electrical Equipment, China University of Mining and Technology, \\ Xuzhou 221116, China \\ Correspondence should be addressed to Wei Li; liwei_cmee@163.com
}

Received 3 September 2018; Revised 24 October 2018; Accepted 31 October 2018; Published 2 December 2018

Academic Editor: Enrico Zappino

Copyright (c) 2018 Mingquan Qiu et al. This is an open access article distributed under the Creative Commons Attribution License, which permits unrestricted use, distribution, and reproduction in any medium, provided the original work is properly cited.

In order to diagnose bearing faults under different operating state and limited sample condition, a fault diagnosis method based on adjusted spectrum image of vibration signal is proposed in this paper. Firstly, the Davies-Bouldin index (DBI) is employed to select a proper capture focus (CF) and image size, and the spectrum of vibration signal is computed via fast Fourier transformation (FFT) and adjusted according to the average rotating speed. Then, the spectrum is plotted and captured as a two-dimensional (2D) image with the optimized CF and image size. Two-dimensional principal component analysis (2DPCA) is used to reduce the dimension of images, and finally a nearest neighbour method is applied to classify the faults of bearings. Two experiments are carried out to validate the effectiveness of the proposed method. Besides, a further investigation on the effect of spectrum frequency resolution is conducted and a recommended selection method of frequency resolution is given based on the experimental performances. In our method, the training samples could be from only one operating condition, while the testing samples are from all possible operation conditions. All experiment results have demonstrated that the proposed method could achieve high classification accuracy even with very limited training samples.

\section{Introduction}

As the key element of rotating machinery, the faults of rolling bearing could lead to mechanical breakdown and great economic loss. In general, the operating speed of rolling bearing is slightly fluctuant due to the influences from the load, controller, and other components; therefore, its vibration signals are commonly considered approximately stationary. However, rolling bearing may operate in different speeds, which results in great challenges for its accurate fault diagnosis, especially when the number of fault samples is small. Most fault diagnosis methods are based on vibration signals [1-3], and the diagnostic procedure mainly includes two steps: (1) extracting features from the vibration signal; and (2) classifying features with a classifier. In last decades, many methods were proposed to improve the diagnosis accuracy in two different ways. One is to find proper features in order to represent the characteristics of faulty vibration signals; and the other one is to find proper classifiers with strong classification capability.

The features could be extracted in time domain, frequency domain, or time-frequency domain [4], such as peak amplitude, skewness, kurtosis, fractal dimension, Fourier spectrum, cepstrum, and envelope spectrum. Images were also utilized as features for machine fault diagnosis in recent years. For example, Li et al. [5] proposed a method of feature extraction with spectrum image for bearing fault diagnosis. Amar et al. [6] proposed to use vibration spectrum imaging feature for denoising and fault classification. Klein et al. [7] utilized time-frequency image of vibration signal to detect fault signatures with an object detection method. Griffaton et al. [8] realized bearing damage detection of aircraft engines through enhanced visual analysis with the help of image processing method. This feature expression of image for one-dimensional (1D) vector seems to be a very promising way to dig the intrinsic information contained in the vibration signal. 
Once the fault features are extracted, the last step is to perform the fault classification to recognize the corresponding health state. Fault classification can be realized with minimum distance classifier, $\mathrm{k}$-nearest neighbour (kNN) classifier, artificial neural network (ANN), support vector machine (SVM), and so on [4]. With proper features, those classifiers may achieve acceptable accuracy of diagnosis. When noise is heavy or the number of fault samples is small, the accuracy may decrease significantly. Recently, deep neural networks (DNNs) were applied for fault diagnosis of rotating machine [9-11]. Such DNNs can extract useful features from some raw information and achieve high classification accuracy. Nevertheless, DNNs usually require a high number of training samples and high computation efforts. When the operating conditions are different, the training samples under all operating conditions may be required, and the training effort could be extremely high. Therefore, a robust method of feature extraction or pattern classification needs to be further investigated to improve the performance on fault diagnosis.

Focusing on extracting a more effective feature, we proposed to conduct bearing fault diagnosis with adjusted vibration spectrum images. This work is an extension of the spectrum images proposed in our previous publication [5], where spectrum images are first proposed as features for bearing fault diagnosis and preferable diagnostic effects are reached especially in the case of acquiring samples under the same working condition. However, it exhibits a poor performance when the training and testing samples are derived from different operating conditions (see the results marked with $\star$ in [5]) due to the operating-condition difference and the nonoptimized parameters such as frequency resolution, image size, and capture focus of the spectrum images. Driven by the desire to extract a more robust feature for different operating conditions, an improved fault diagnosis method with adjusted spectrum images is proposed in this paper. We consider a more difficult situation: (1) the operating condition could be different, i.e., the rotating speed and/or load is different; (2) the number of training samples is very limited, and the training samples could be from only one operating condition. Firstly, a validity metric is introduced to optimally select the parameters for generating images, and the spectrum of vibration signal is computed with the help of the fast Fourier transformation (FFT). Secondly, the frequency lines of the spectrum are adjusted according to the rotating speed frequency to reduce its influence on the spectrum image. Thirdly, such adjusted spectrum is captured as a two-dimensional (2D) image with the optimized parameters and then processed through two-dimensional principal component analysis (2DPCA) to reduce its dimension. Finally, fault classification is achieved with a simple nearest neighbour classifier (NNC). Experimental examples are applied to demonstrate the effectiveness of the proposed method. And it is shown that classification accuracy using such features is higher than that obtained by directly using the FFT spectrum.

The main contributions of this paper can be concluded as follows: (1) the influence of operating conditions (especially for the rotating speed) on vibration spectrum is reduced through rotating-speed-adjusting manipulation; (2) the adjusted vibration spectrum with optimized parameters is captured as image feature to perform bearing fault diagnosis; (3) higher classification accuracy can be achieved by serving such adjusted spectrum images as features even in the case where simple classifier and very limited samples are employed especially under different operating conditions.

The paper is organized in the following way. Section 2 presents the generation of adjusted spectrum images, and the parameter determination for generating images is depicted in Section 3. Section 4 is dedicated to illustrating 2DPCA and classification measure, including feature processing with 2DPCA and fault classification based on NNC. Section 5 provides the experimental analysis and discussion is given in Section 6. Finally, Section 7 presents the conclusions.

\section{Generation of Adjusted Spectrum Images}

The spectrum of vibration signal can be computed through FFT. Such a spectrum contains lots of useful information of bearing, and the vector of spectrum coefficients is widely used as features for fault diagnosis $[4,9]$. As we know, the bearing characteristic frequencies are associated with the rotating speed. As a result, the frequency lines corresponding to those characteristic frequencies and their harmonics could be dominant components of the spectrum. When the rotating speed is different, those dominant frequency components are also changing with the speed. In this case, bearing diagnosis becomes difficult. Hence, we propose to use the adjusted spectrum image as feature for fault diagnosis instead of the spectrum coefficient vector.

Firstly, the spectrum of vibration signal is computed via FFT. We plot the spectrum as a diagram, where the $x$-axis is the frequency and the $y$-axis is the amplitude. As discussed before when the speed of bearing is changing, the structure of the diagram is also changing. It is reasonable to adjust all frequency lines of the spectrum according to the rotating speed such that the change of diagram structure can be reduced, i.e.,

$$
F_{\mathrm{a}}(k)=\frac{F(k)}{f_{\mathrm{r}}},
$$

where $f_{\mathrm{r}}$ is the average rotating speed in $\mathrm{Hz}, F(k)$ is the $k$ th frequency line of the original spectrum, and $F_{\mathrm{a}}(k)$ is the $k$ th frequency line of the adjusted spectrum.

After adjusting, the spectrum diagram can be captured as an image with a given size. Two parameters of the image are selectable, i.e., the coordinate range and image size when storing. The coordinate range includes the range of the $x$-axis $\left(R_{x}\right)$ and the range of the $y$-axis $\left(R_{y}\right) . R_{x}$ determines the frequency range shown in the image, and $R_{y}$ determines the amplitude range shown in the image. We call $R_{x}$ and $R_{y}$ together as the capture focus (CF). Later, we will show the selection method of CF and image size.

The spectrum is actually a $1 \mathrm{D}$ vector of FFT coefficients, while the spectrum image is a $2 \mathrm{D}$ representation of the $1 \mathrm{D}$ vector. Such images can keep the geometric information of frequency lines and provide richer information for fault diagnosis than the $1 \mathrm{D}$ vector of spectrum. By adjusting the 
spectrum according to the rotation speed, the obtained images are robust against speed variations. In order to demonstrate the effectiveness of the proposed adjusted spectrum images in suppressing the interference of rotating speed and reducing the changes of diagram structure, a comparison is conducted between before and after spectrum adjusting using vibration signals collected from the drive end bearing with inner-race fault size being 0.021 inches under two speed conditions [12]. The analyzed signals both have a length of 1024 data points in time domain. Moreover, they are both transformed into frequency domain through $\mathrm{N}$-point discrete Fourier transform (DFT), where $\mathrm{N}$ is derived as the next power of two from the length of the time series, and $N=1024$ as well. For simplicity, the coordinate system are not hid to present the role of the spectrum image adjusting, and the $\mathrm{CF}$ of the original and adjusted spectrum are set as $R_{x}=[06000], R_{y}=\left[\begin{array}{ll}0 & 0.2]\end{array}\right.$ and $R_{x}=\left[\begin{array}{ll}0 & 210\end{array}\right], R_{y}=$ $\left[\begin{array}{ll}0 & 0.2]\end{array}\right]$, respectively.

To be clear, the spectra's places may influence diagnostic performance when the spectrum plot, in fact a 2D matrix, is captured as feature for fault diagnosis. Hence, we construct an index to evaluate the performance of adjusted spectrum as follows:

$$
H=\frac{\left|S^{\left\{f_{1}\right\}}-S^{\left\{f_{2}\right\}}\right|}{s_{\mathrm{r}}},
$$

where $|\cdot|$ stands for calculating absolute value, $S\left\{f_{1}\right\}$ and $S\left\{f_{2}\right\}$ denote the area between the Fourier transform curve and the horizontal axis (frequency axis) under rotating speed frequency $f_{1}$ and $f_{2}$, respectively, and $s_{r}$ is the spectrum scaling factor for horizontal axis, i.e., $s_{r}=6000$ for the original spectrum and $s_{r}=210$ for the adjusted one here. Obviously, a smaller value of $H$ indicates a better similarity between two curves from the view of image.

As shown in Figure 1(a), the frequency spectrum may mismatch due to the variation of rotating speed. However, the adjusted frequency is employed to plot the spectrum in Figure 1(b). In this process, the spectrum line under the same order of different rotating frequencies is placed in the same position of the adjusted spectrum plot. Obviously, a smaller $H$ represents a better case for fault diagnosis, for the original spectrum, $H=0.0013$, and for the adjusted spectrum, $H=3.8236 \times 10^{-6}$. It is clear that the adjusted images could decouple the rotating speed for the similar health condition of machines. This adjusted spectrum obtained as image shows its stronger robustness to speed variations thanks to the adjusting manipulation. It will be demonstrated by the subsequent experiments.

\section{Determination of CF and Image Size}

The main concept of our approach is to catch the structural information of spectrum to perform bearing fault diagnosis. A vibration spectrum image with different sizes may contain different information of the spectrum structure. Generally, the larger a spectrum image is, the more abundant information it carries within a certain range. However, a larger image could consume more storage space and increase the influence of noise. Moreover, different CFs can mostly capture different levels of information in the adjusted spectrum images, and a CF specifies the window for capturing images to define the feature contents. Therefore, it is crucial to determine these two parameters when applying to bearing fault diagnosis.

In this research, a validity indicator, Davies-Bouldin index (DBI) proposed in [13], is employed to evaluate the image size and the CF for capturing adjusted spectrum images. The DBI is defined as the ratio of within-class scatter to between-class scatter and its calculating process can be concluded as follows:

Firstly, the within-class scatter for each cluster (i.e., the fault category in this research) is calculated as

$$
S_{i}=\left(\frac{1}{T_{i}} \sum_{j=1}^{T_{i}}\left|X_{j}-A_{i}\right|^{p}\right)^{1 / p},
$$

where $A_{i}$ is the centroid of the $i$ th cluster, $T_{i}$ is the number of data samples in $i$ th cluster, $X_{j}$ represents the $j$ th data sample in $i$ th cluster, and $p$ is generally set as 2 in real applications.

Secondly, the between-class scatter for two different clusters is computed as

$$
M_{i j}=\left\|A_{i}-A_{j}\right\|^{p}=\left(\sum_{k=1}^{n}\left|a_{k i}-a_{k j}\right|^{p}\right)^{1 / p},
$$

where $a_{k i}$ and $a_{k j}$ are the column vector of $A_{i}$ and $A_{j}$, respectively.

Finally, the so-called DBI is denoted as

$$
\mathrm{DBI}=\frac{1}{N_{\mathrm{b}}} \sum_{i=1}^{N_{\mathrm{b}}} D_{i}
$$

where $N_{\mathrm{b}}$ is the number of clusters, and $D_{i}=\max _{j \neq i}$ $\left(S_{i}+S_{j} / M_{i j}\right)$.

From the calculation process of the DBI, it is not hard to find that a image size or CF for capturing adjusted spectrum images with a smaller DBI could be beneficial to achieving better classification.

\section{Feature Processing and Classification}

Since the size of adjusted spectrum images is large, the computation effort could be considerably high if we carry out diagnosis by directly using those images. Hence, the adjusted spectrum images are firstly processed by 2DPCA in order to reduce their dimension and then classified with the NNC.

4.1. Feature Processing with 2DPCA. Two-dimensional principal component analysis (2DPCA) is a dimension reduction method for image representation. In 2DPCA, the image covariance matrix is constructed directly using the original image matrices, and its eigenvectors are derived for image feature extraction. The principle of 2DPCA can be found in [14].

As illustrated in Figure 2, $M$ adjusted spectrum images are firstly obtained with the size of $w \times h$ pixels. Then, the global image scatter matrix $G$ is evaluated according to 


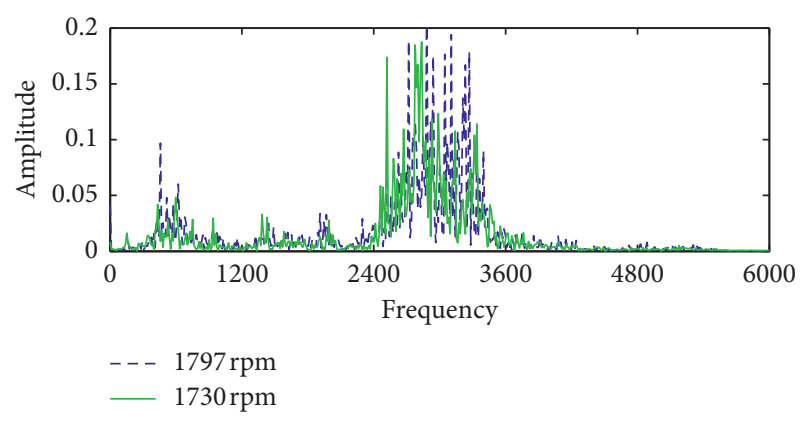

(a)

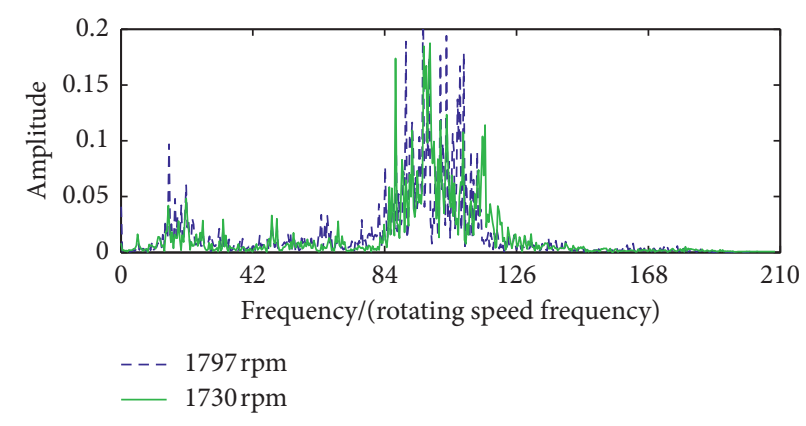

(b)

Figure 1: A comparison of spectrum image of before and after adjusting: (a) the original spectrum, (b) the adjusted spectrum.

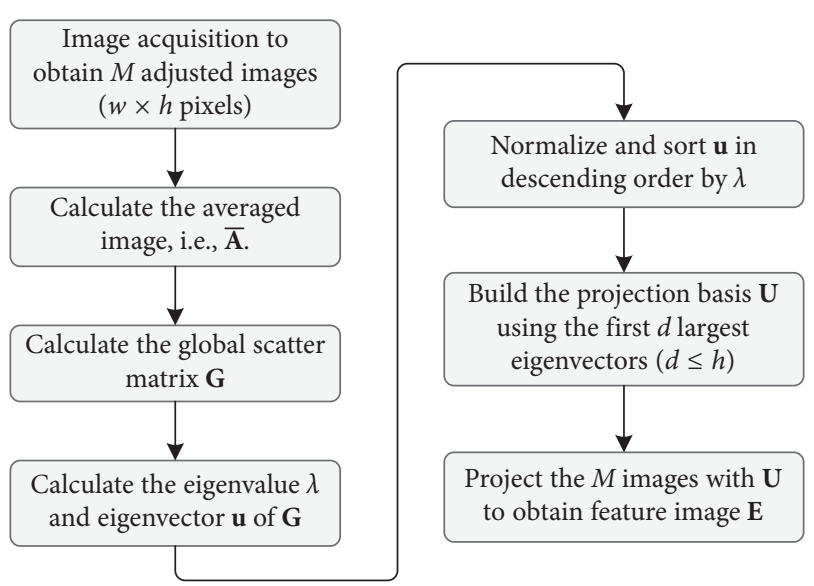

FIGURE 2: Flowchart of feature processing with 2DPCA.

$$
G=\frac{1}{M} \sum_{j=1}^{M}\left(\mathbf{A}_{j}-\overline{\mathbf{A}}\right)^{T}\left(\mathbf{A}_{j}-\overline{\mathbf{A}}\right) \in R^{h \times h},
$$

where $\mathbf{A}_{j}(j=1,2, \ldots, M)$ is the $j$ th image, and $\overline{\mathbf{A}}$ is the averaged image of all the $M$ images, i.e., $\overline{\mathbf{A}}=(1 / M) \sum_{j=1}^{M} \mathbf{A}_{j}$.

Secondly, the eigenvalue $\lambda_{k}$ and the corresponding eigenvector $u_{k}$ of $G$ are calculated. Then $u_{k}$ is normalized and sorted in descending order by $\lambda_{k}$. That is, $u=$ $\left(u_{\max 1}, u_{\max 2}, \ldots, u_{\max h}\right)$, and the corresponding eigenvalue is $\lambda=\left(\lambda_{\max 1}, \lambda_{\max 2}, \ldots, \lambda_{\max h}\right)$, where

$$
\lambda_{\max 1}>\lambda_{\max 2}>\ldots>\lambda_{\max h} .
$$

The first $d(d \leq h)$ largest eigenvalues are selected according to Equation (8):

$$
d=\min _{d}\left(\frac{\sum_{j=1}^{d} \lambda_{\max j}}{\sum_{i=1}^{h} \lambda_{\max i}} \geq \gamma\right),
$$

where $\gamma$ is called eigenvalue contribution and $0<\gamma \leq 1$. Then, the projection basis is constructed as

$$
\mathbf{U}=\left[\begin{array}{llll}
u_{\max 1} & u_{\max 2} & \cdots & u_{\max d}
\end{array}\right] .
$$

Finally, all the $M$ samples are projected into the so-called eigenspace, and the corresponding eigenimages are obtained. Given an adjusted spectrum image $B$, it can be projected according to

$$
Y_{k}=\mathbf{B} U_{k}, \quad k=1,2, \ldots, d,
$$

where $U_{k}=u_{\max k}$. Then the eigenimage of $B$ is obtained as $\mathbf{E}=\left[Y_{1}, Y_{2}, \ldots, Y_{d}\right]$.

4.2. Classification Based on NNC. The projected eigenimage with reduced dimension will be obtained after an adjusted image is processed with 2DPCA. Then the following NNC [15] is applied for fault classification.

Suppose that there are $M$ training images being categorized into $N_{\mathrm{c}}$ classes and the classification labels are defined as

$$
s_{1}, s_{2}, \ldots, s_{N_{c}} \text {. }
$$

Denote $E$ as the eigenimage of a testing image $F$, and $\mathbf{E}_{i}$ is the eigenimage of the $i$ th training image. The distance function $D_{i}(\mathbf{F})$ of $F$ is defined as

$$
D_{i}(\mathbf{F})=D\left(\mathbf{E}, \mathbf{E}_{i}\right)=\sum_{r=1}^{d}\left\|Y_{r}^{(\mathbf{F})}-Y_{r}^{(i)}\right\|_{2}, \quad i=1,2, \ldots, M,
$$

where $\left\|Y_{r}^{(\mathbf{F})}-Y_{r}^{(i)}\right\|_{2}$ denotes the Euclidean distance between $Y_{r}^{(\mathbf{F})}$ and $Y_{r}^{(i)}$, and $Y_{r}^{(\mathbf{F})}$ represents the $r$ th vector of matrix $E$, and $Y_{r}^{(i)}$ represents the $r$ th vector of matrix $\mathbf{E}_{i}$.

Then the decision-making process is carried out according to

$$
D_{j}(\mathbf{F})=\min _{i} D_{i}(\mathbf{F}),
$$

where $j$ means the $j$ th training image. If the $j$ th training image belongs to the $s_{q}$ th class $\left(q \in\left\{1,2, \ldots, N_{c}\right\}\right)$, then the classification label of the testing image $F$ is determined as $s_{\mathrm{q}}$. In fact, the testing image is classified according to the nearest training image, where the Euclidean distance is used.

Other classifiers, such as ANN and SVM, can also be applied to classify the dimension-reduced features, but the model parameters are hard to optimize with limited training samples during the training process. However, the NNC is without training procedure for model-parameter determination during fault classification. Hence, selecting NNC as the classifier for fault diagnosis in this work mainly takes account of the computational efficiency and the limited sample condition.

Therefore, the main procedure of the proposed fault diagnosis scheme can be described as follows: 
(i) The vibration signals are first converted into adjusted spectrum images via FFT according to Sections 2 and 3

(ii) Then the training images are used to calculate the projection basis with 2DPCA according to Equations (6), (8), and (9), and all images are processed with the derived projection basis to obtain the eigenimages according to Equation (10)

(iii) Finally, the testing images are classified through NNC according to Equations (12) and (13)

\section{Experimental Analysis}

The proposed adjusted spectrum image can be widely used in diagnosis of bearing, including fault type classification and fault severity detection. Two experiments are carried out to demonstrate the effectiveness of the proposed method. In the first one, different types of bearing faults are classified. In the second one, single-fault and multifault severity diagnosis of bearings are conducted, where the rotating speed and the load are different. The results of the second experiment are also comprehensively compared with that in previous publications.

\subsection{Experiment I: Fault Type Classification of Bearings}

5.1.1. Experimental Setup. The first experiment is performed on a machinery fault simulator manufactured by Spectra Quest, Inc. shown in Figure 3. The simulator is a simplified version of complex rotating machines, which consists of motor, bearing, shaft, rotor disc, gearbox, and belt. In this experiment, a normal bearing was installed in the inboard bearing seat and the testing bearings were mounted on the outboard bearing seat in turn, which simulate following health conditions: (i) normal (NO), (ii) inner-race fault (IF), (iii) outer-race fault (OF), and (iv) ball fault $(\mathrm{BF})$. Figure 4 presents the bearing fault conditions. All the bearings are the same type ER-12k with 8 rolling elements. Vibration signals were collected under three operating conditions, i.e., $600 \mathrm{rpm}, 900 \mathrm{rpm}$, and $1200 \mathrm{rpm}$. And the sampling frequency was set to $20 \mathrm{kHz}$.

The vibration signals are organized as three datasets according to the operating conditions, i.e., A-600 rpm, B-900 rpm, and C-1200 rpm as described in Table 1. Every dataset contains 300 vibration signal samples from each health condition. Hence, every dataset consists of 1200 samples. The length of each sample is 1024 points in time domain, and each time series is converted into frequency domain via $N$-point DFT ( $N$ is derived as the next power of two from the length of the time series, so $N=1024$ as well) to generate its adjusted spectrum image. The number of classification labels is four, where each label is corresponding to a health condition of bearings.

5.1.2. Parameters Determination. The CF selection is first considered with the DBI evaluation approach. In this study, the $R_{x}$ in CF is manually designated to guarantee that the derived images could contain all the adjusted

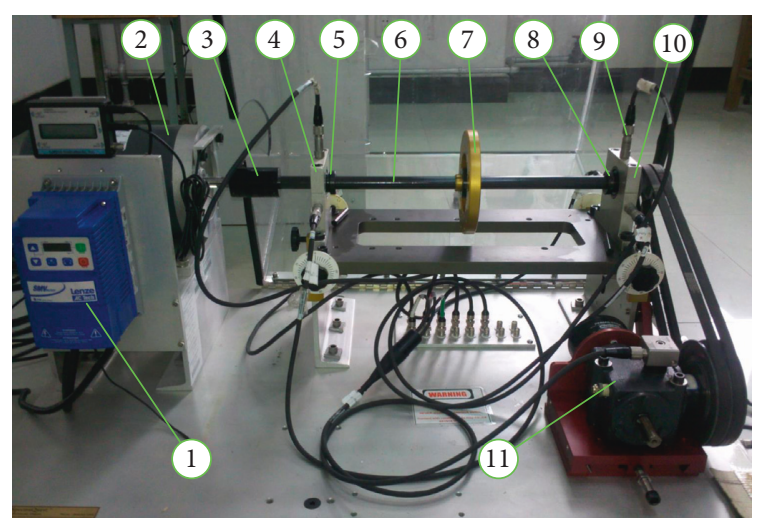

Figure 3: Machinery fault simulator: 1, frequency converter; 2, motor; 3, coupling; 4 , inboard bearing seat; 5 , normal bearing; 6 , shaft; 7, rotor disc; 8 , testing bearing; 9 , acceleration sensor; 10 , outboard bearing seat; 11, gearbox.

frequency components, i.e., $R_{x} \geq(10000 / 10)=1000$ for dataset $\mathrm{A}, R_{x} \geq(10000 / 15)=666.67$ for dataset $\mathrm{B}$, and $R_{x} \geq(10000 / 20)=500$ for dataset $C$ according to the sampling rate $\left(F_{\mathrm{s}}=20000 \mathrm{~Hz}\right)$. Therefore, the $R_{x}$ of dataset $\mathrm{A}$ is set as $R_{x}=$ [0 1000], the $R_{x}$ of dataset $\mathrm{B}$ is set as $R_{x}=$ [0 670], and the $R_{x}$ of dataset $\mathrm{C}$ is set as $R_{x}=[0500]$.

In order to optimize a proper $R_{y}=\left[0 y_{\mathrm{cf}}\right]$ in $\mathrm{CF}$ to generate the final adjusted spectrum images, data samples from dataset A are utilized to calculate the DBI for four bearing health conditions. Here, the image size with $400 \times$ 400 pixels is preassigned empirically according to our experimental experiences. In view of the enormous computing effort for DBI evaluations, a scope of $y_{\mathrm{cf}}$ from 0.0005 to 0.2 (this range is determined with experience) covering $12 \mathrm{CFs}$ are considered to select an appropriate CF with the assistance of DBI evaluations, i.e.,

$$
\begin{aligned}
& y_{\mathrm{cf}} \in\{0.2,0.17,0.14,0.1,0.08,0.06,0.05,0.04,0.02, \\
&0.01,0.001,0.0005\},
\end{aligned}
$$

and different training sizes of each health state $\left(n_{\mathrm{dbi}}\right)$ are employed for the DBI assessment. The DBI values for different CFs are presented in Figure 5. It can be found that the DBI reaches a minimum at the 9 th $y_{\mathrm{cf}}$, i.e., $y_{\mathrm{cf}}=0.02$. In this experiment, the $R_{y}$ of CF is set to $R_{y}=\left[\begin{array}{ll}0 & 0.02\end{array}\right]$ for datasets $\mathrm{A}, \mathrm{B}$, and $\mathrm{C}$.

As what considered above, a scope of image size from $[120 \times 120]$ to $[700 \times 700]$ pixels are taken into account with experimental experience and 12 image sizes are applied to test on dataset A with the derived CF to select an appropriate one, i.e., $r_{\mathrm{s}} \times[100 \times 100]$ pixels, where

$$
r_{\mathrm{s}} \in\{1.2,1.5,2,2.5,3,3.5,4,4.5,5,5.5,6,7\} \text {. }
$$

Also considering different kinds of image samples, the DBI values are depicted in Figure 6. It can be shown that an optimized image size is achieved at the 6th testing size, i.e., $[350 \times 350]$ pixels.

Thereafter, the spectrum is obtained for each sample through FFT and adjusted according to Equation (1). The CF of dataset $\mathrm{A}$ is set as $R_{x}=[01000], R_{y}=[00.02]$, the CF of dataset $\mathrm{B}$ is set as $R_{x}=\left[\begin{array}{ll}0670\end{array}\right], R_{y}=\left[\begin{array}{ll}0 & 0.02\end{array}\right]$, and 


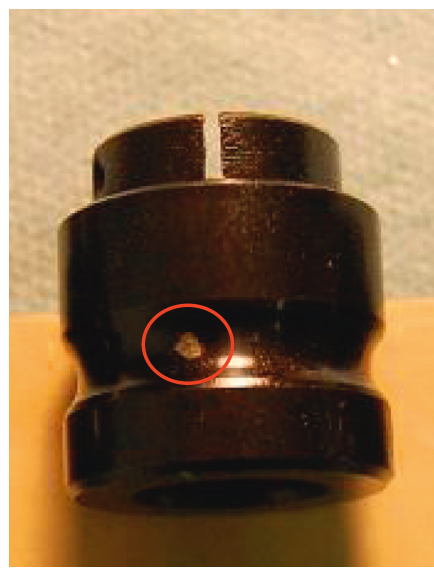

(a)

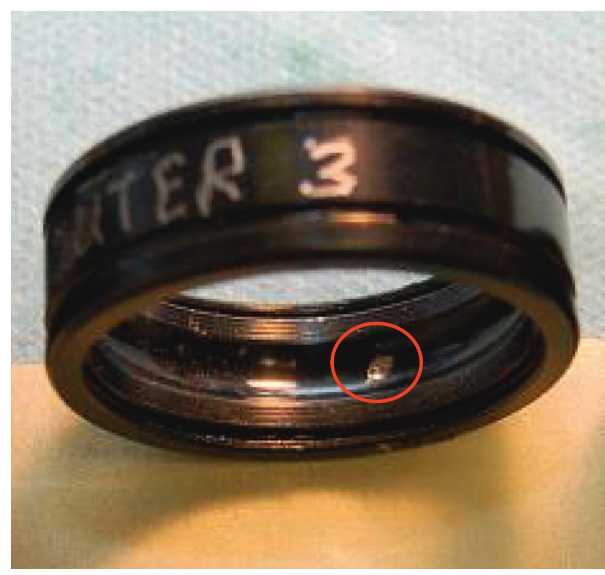

(b)

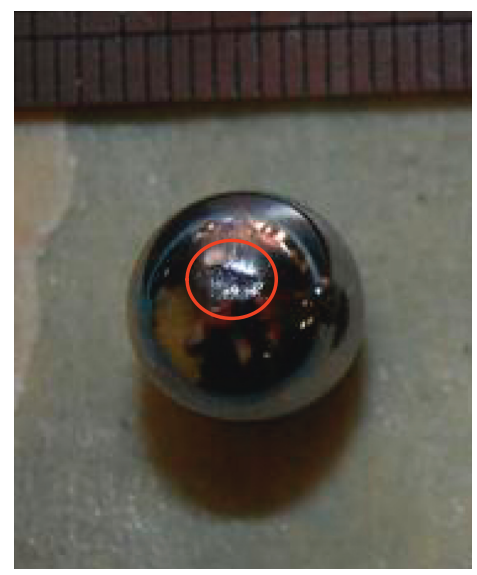

(c)

Figure 4: Pictures of the bearing faults: (a) IF; (b) OF; (c) BF.

TABLE 1: Description of bearing datasets for fault classification.

\begin{tabular}{|c|c|c|c|c|}
\hline Dataset & $\begin{array}{l}\text { Speed } \\
(\mathrm{rpm})\end{array}$ & $\begin{array}{c}\text { No. of } \\
\text { samples }\end{array}$ & $\begin{array}{l}\text { Health } \\
\text { condition }\end{array}$ & $\begin{array}{c}\text { Classification } \\
\text { label }\end{array}$ \\
\hline \multirow{4}{*}{$\mathrm{A} / \mathrm{B} / \mathrm{C}$} & \multirow{4}{*}{$\begin{array}{c}600 / 900 / \\
1200\end{array}$} & $\begin{array}{c}300 / 300 / \\
300\end{array}$ & $\mathrm{NO}$ & 1 \\
\hline & & $\begin{array}{c}300 / 300 / \\
300\end{array}$ & IF & 2 \\
\hline & & $\begin{array}{c}300 / 300 / \\
300\end{array}$ & OF & 3 \\
\hline & & $\begin{array}{c}300 / 300 / \\
300\end{array}$ & $\mathrm{BF}$ & 4 \\
\hline
\end{tabular}

the CF of dataset $\mathrm{C}$ is set as $R_{x}=\left[\begin{array}{l}0 \\ 500\end{array}\right], R_{y}=\left[\begin{array}{ll}0 & 0.02\end{array}\right]$. Finally, all the adjusted spectrum images are captured with the size being $350 \times 350$ pixels. Figures 7-9 show the adjusted spectrum images of dataset $A, B$, and $C$, respectively.

5.1.3. Experimental Results. After obtaining the adjusted spectrum images using the optimally selected parameters, feature extraction is carried out with 2DPCA to derive the eigenimages of the adjusted spectrum images. Herein, the dataset $\mathrm{A}$ is employed to demonstrate the eigenimages in the phase of feature processing, where the training size of each class is set as 20 and the reduced dimension in Equation (8) is set as $d=50$ manually. Figure 10 shows an example of the eigenimages of dataset $\mathrm{A}$.

With $\gamma=0.9$ defined in Equation (8) for dimension reduction, the diagnosis performance using the proposed method is tested and shown in Table 2, where different number of samples are used for training the NNC and the average accuracies are presented for 20 randomized trials. Obviously, the classification accuracy reaches $100 \%$ with 20 images per class for training, which means that all the health conditions can be correctly classified in these cases. Even when only one sample is used for training, high classification accuracies are still achieved.

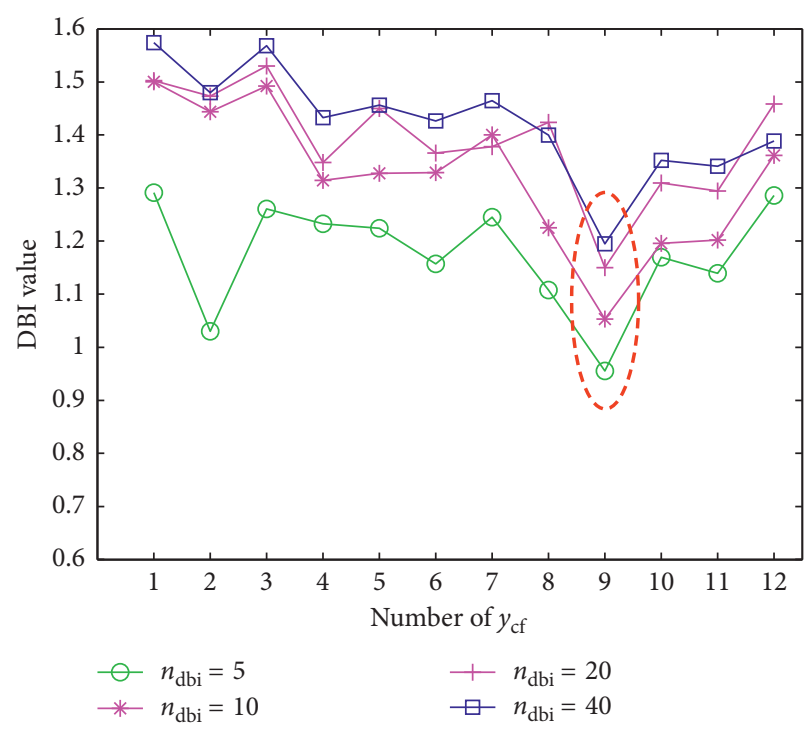

Figure 5: Experiment I: the DBI values for $R_{x}=[01000]$ and $R_{y}=\left[\begin{array}{ll}0 & y_{\mathrm{cf}}\end{array}\right]$.

\subsection{Experiment II: Fault Severity Detection of Bearings}

5.2.1. Data Description. In order to further verify the effectiveness of the proposed method, vibration data collected from the bearing data centre of Case Western Reserve University (CWRU) [12] are also applied. The bearing vibration signals under four health conditions are used for evaluation, i.e., NO, IF, BF, and $\mathrm{OF}$, which are the same with experiment $\mathrm{I}$. The sampling rate is $12 \mathrm{kHz}$. IF, BF, and OF of bearings are with four different fault severities, i.e., 0, 0.007, 0.014 , and 0.021 inches. Four load conditions with different rotating speeds are considered, i.e., Load $0=0 \mathrm{hp} / 1797 \mathrm{rpm}$, Load $1=1 \mathrm{hp} / 1772 \mathrm{rpm}$, Load $2=2 \mathrm{hp} / 1750 \mathrm{rpm}$, and Load $3=3 \mathrm{hp} / 1730 \mathrm{rpm}$, which indicate that the operating condition of this experiment is different in speed and load. The collected vibration signals are organized as dataset D, E, $\mathrm{F}$, and $\mathrm{G}$ as shown in Tables 3 and 4 . 


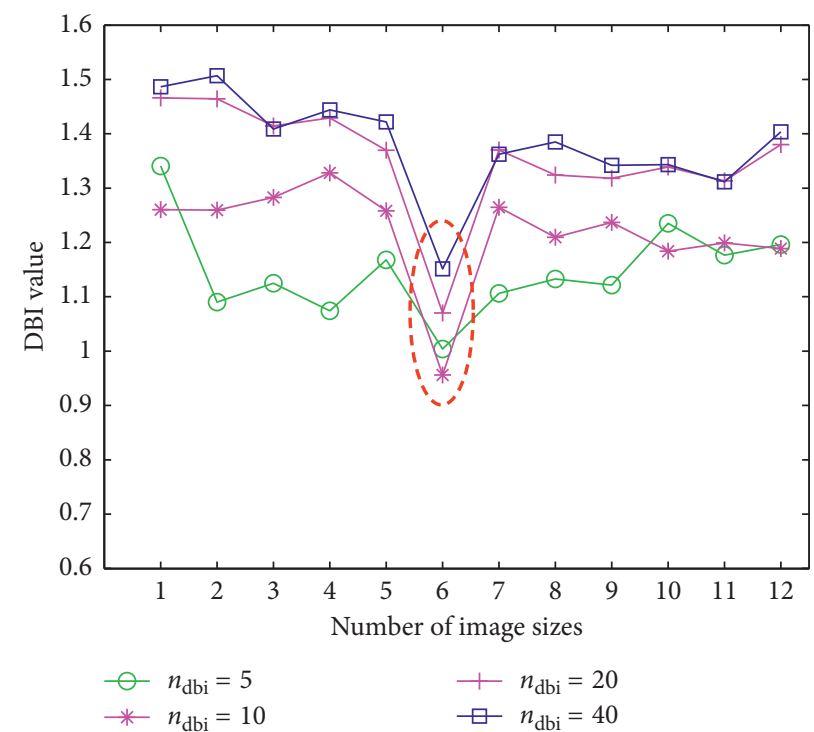

FIGURE 6: Experiment I: the DBI values for different image sizes.

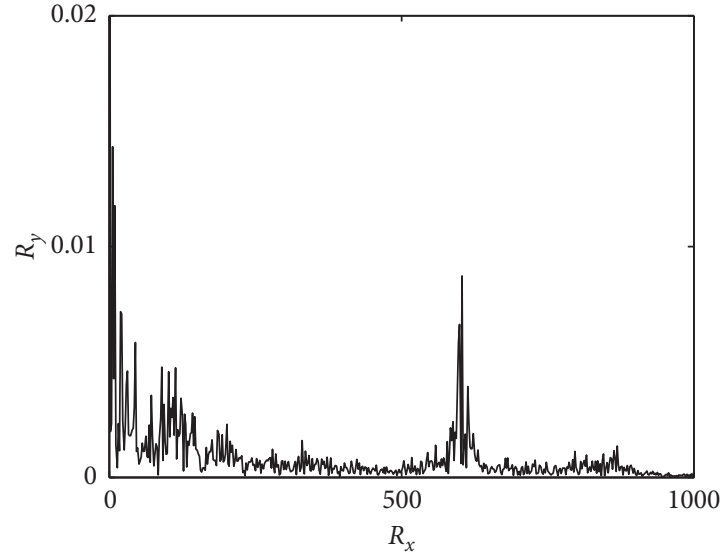

(a)

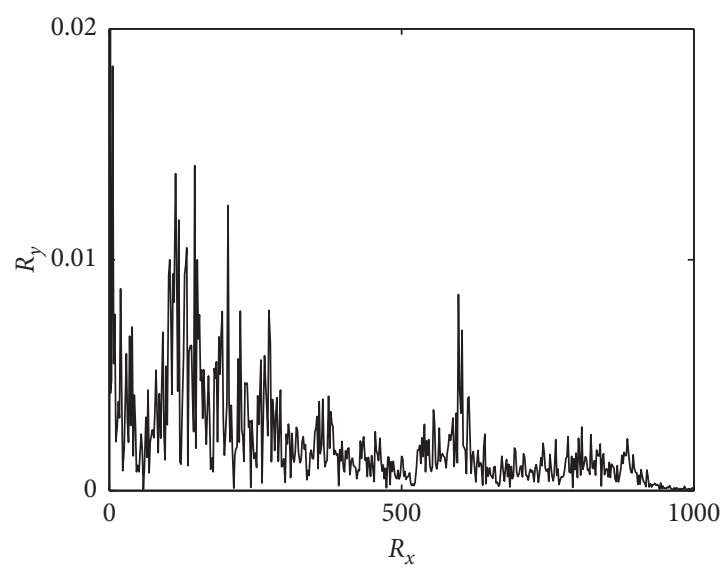

(c)

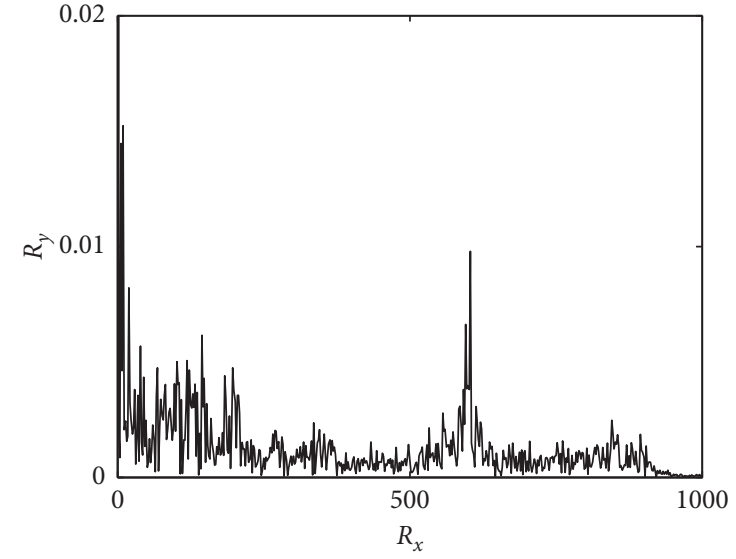

(b)

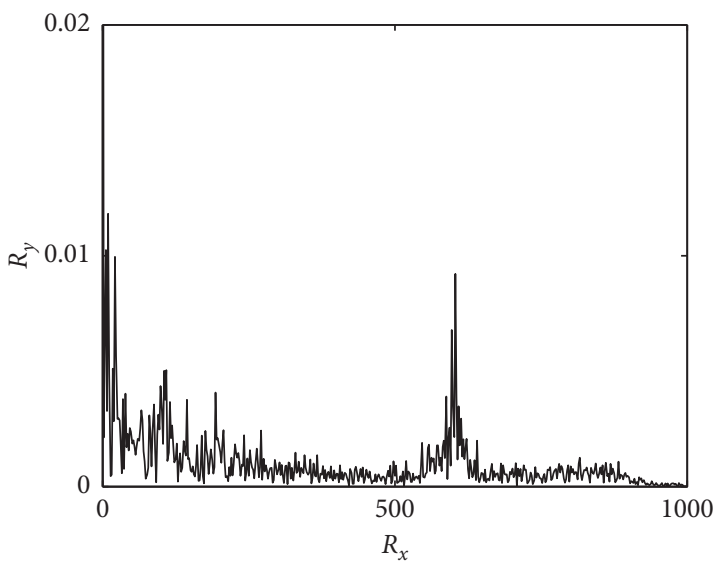

(d)

FIgURE 7: The adjusted spectrum images of dataset A: (a) NO; (b) IF; (c) OF; (d) BF.

Dataset $\mathrm{D}, \mathrm{E}$, and $\mathrm{F}$ are vibration signal samples with IF, BF, and OF, respectively, and they are used for singlefault severity detection. Each dataset consists of four subsets, and each subset is corresponding to a load condition. For instance, D1 is the subset with IF, and its training samples are from Load 0 and its testing samples 


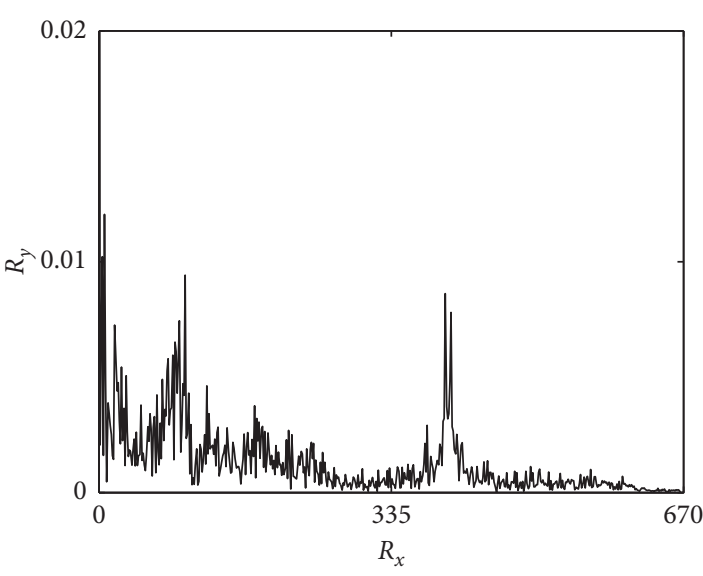

(a)

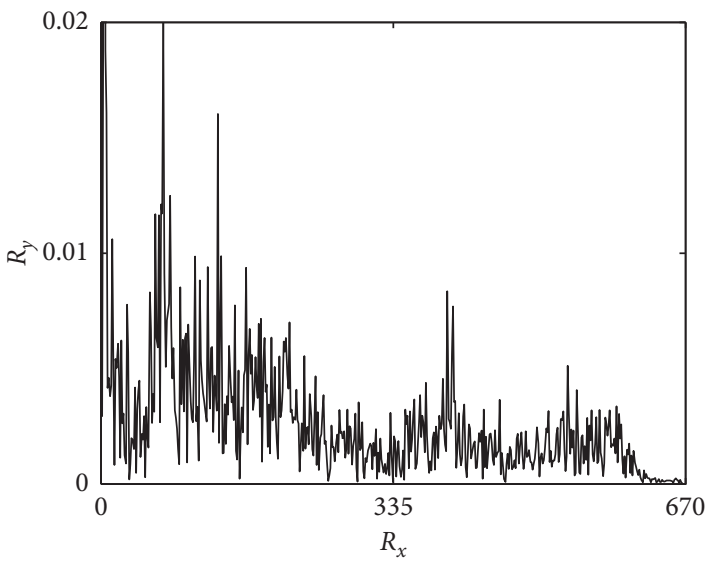

(c)

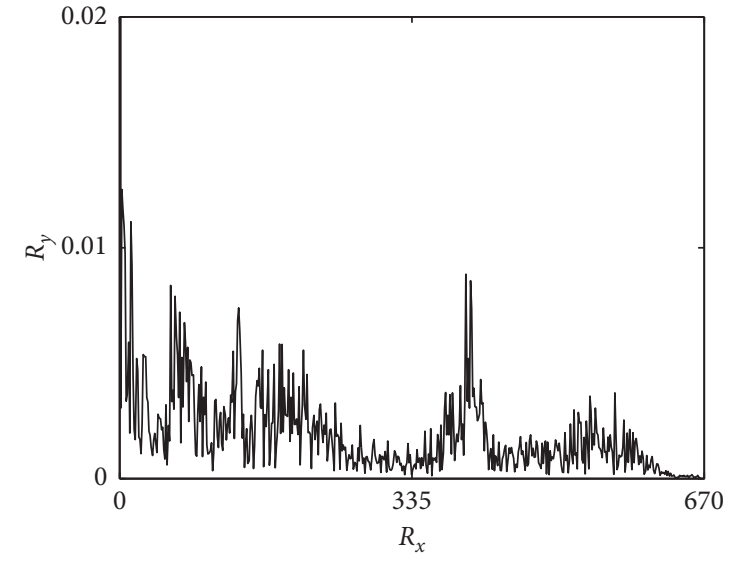

(b)

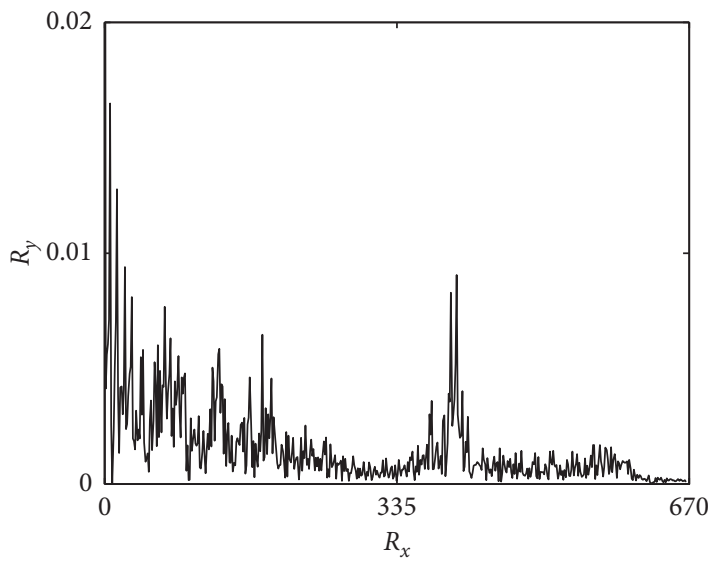

(d)

Figure 8: The adjusted spectrum images of dataset B: (a) NO; (b) IF; (c) OF; (d) BF.

are from all the four load conditions. Subset D1 contains 50 samples of each fault severity, and totally there are 200 samples in D1. The number of classification labels of dataset $\mathrm{D}, \mathrm{E}$, and $\mathrm{F}$ is four, where each label is corresponding to a fault severity.

Dataset $G$ includes all fault types, and it is used for multifault severity detection. It consists of five subsets. G1-G4 are the subsets whose training and testing samples are not exactly from the same load condition. For instance, the training samples of G1 are from Load 0, and the testing samples are from all the four load conditions. Subset G1 contains 50 samples of each fault severity and type. There are 10 different combinations of fault severities and fault types, and therefore there are totally 500 samples in G1. Subset G5 contains 200 samples under each fault severity and type, whose training and testing samples are from all the four load conditions, so a total of 2000 data samples are comprised in G5. Ten classification labels are designated to mark each bearing health condition for this dataset.

5.2.2. Parameters Determination. Taking the abovementioned tests into consideration, we chose the dataset G5 to evaluate the optimized image size and CF for generating the adjusted spectrum images. As for the CF selection, $R_{x}$ is manually set as $R_{x}=[0210]$ to cover the four operating conditions (i.e., 1797, 1772, 1750, and $1730 \mathrm{rpm}$ ) as considered in previous experiment. Similarly, the image size is preset as $400 \times 400$ pixels, then 12 CFs $\left(R_{y}=\left[\begin{array}{ll}0 & y_{\mathrm{cf}}\end{array}\right]\right)$ and four kinds of training size for each class $\left(n_{\mathrm{dbi}}\right)$ are considered to calculate the DBI values, i.e.,

$$
\begin{aligned}
y_{\mathrm{cf}} \in\{0.2,0.17,0.14,0.1,0.08,0.07,0.06,0.05,0.04,0.03, \\
\\
0.01,0.0005\}
\end{aligned}
$$

then the DBI values for different CFs are shown in Figure 11. It can be found that the DBI reaches a minimum at the 10 th $y_{\mathrm{cf}}$, i.e., $y_{\mathrm{cf}}=0.03$.

With the optimized CF for capturing the adjusted spectra, 12 image sizes are utilized to derive an appropriate one with the similar considerations before, i.e., $r_{s} \times[100 \times$ 100] pixels, where

$$
r_{\mathrm{s}} \in\{1.5,2,3,3.5,4,4.5,5,5.5,6,6.5,7,7.5\} .
$$

As presented in Figure 12, it can be shown that a minimum DBI is reached at the 3 rd testing size, i.e., [300 $\times 300]$ pixels.

Thus, the parameters for generating images are optimally obtained by DBI evaluations. In this experiment, 


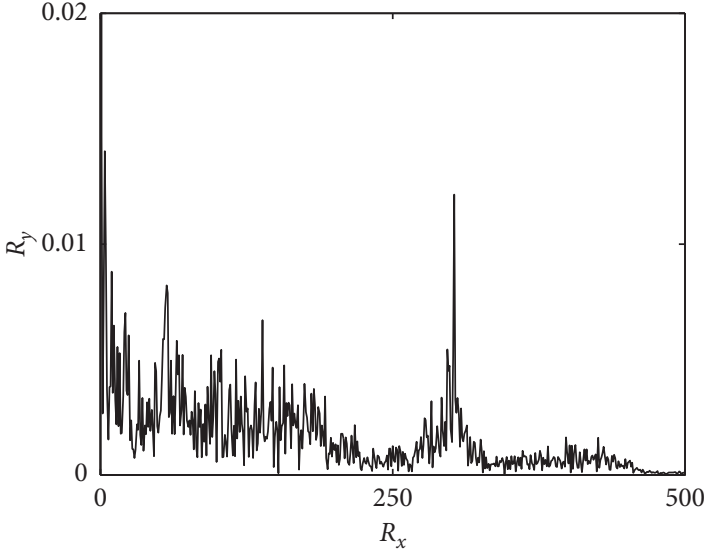

(a)

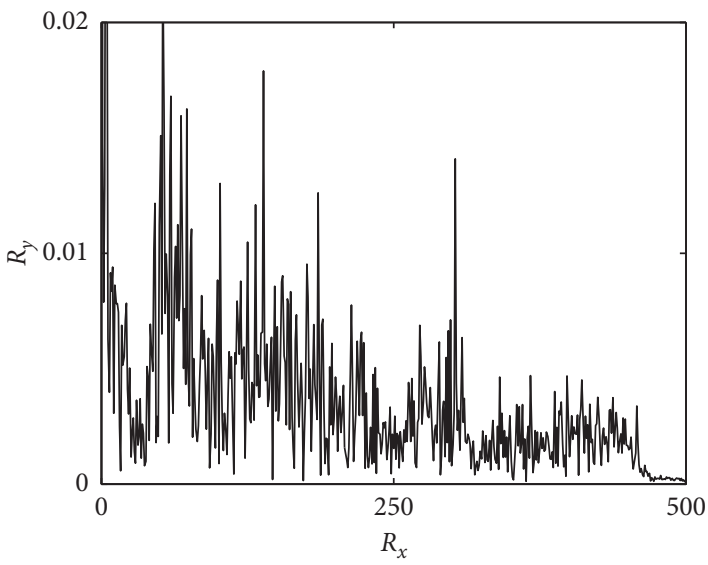

(c)

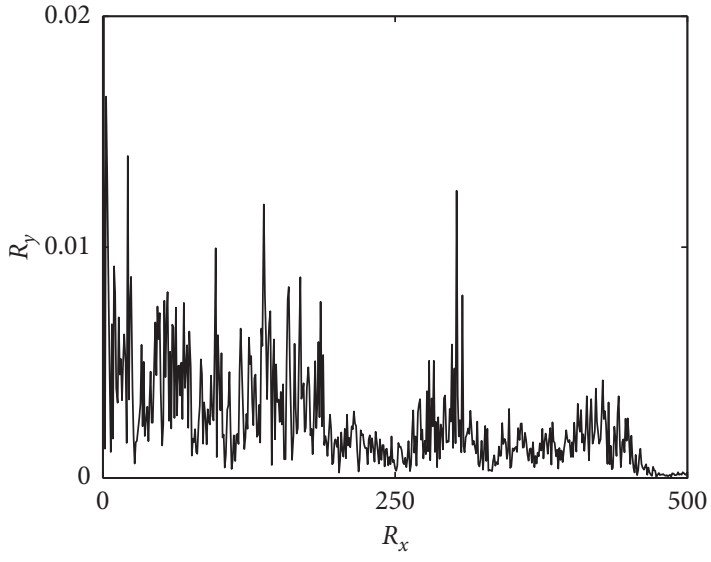

(b)

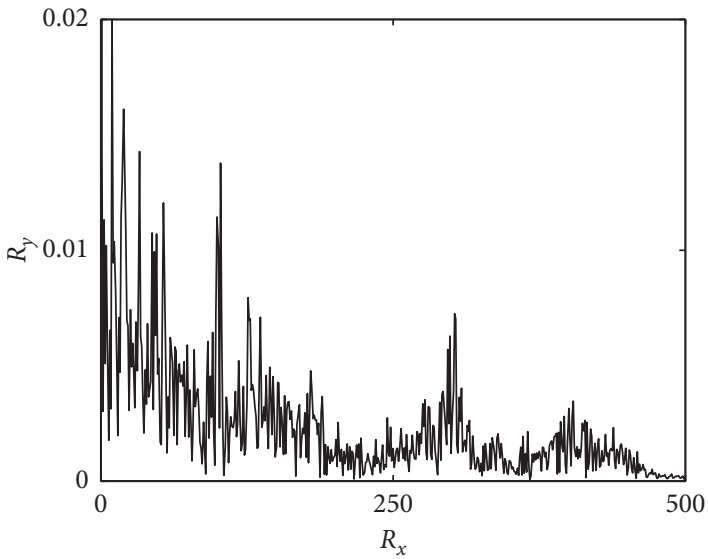

(d)

FIgURE 9: The adjusted spectrum images of dataset C: (a) NO; (b) IF; (c) OF; (d) BF.

each signal segment has a length of 1024 data points in time domain and is also converted to frequency domain using $\mathrm{N}$-point DFT to obtain the corresponding adjusted spectrum image, where $N$ is computed in the same manner as before (in Section 5.1). The adjusted spectrum images are obtained with the size being $300 \times 300$ pixels. Figure 13 gives the adjusted image examples of dataset D1 with the optimized CF $\left(R_{x}=\left[\begin{array}{l}0210\end{array}\right], R_{y}=\left[\begin{array}{ll}0 & 0.03\end{array}\right]\right)$.

5.2.3. Experimental Results. Likewise, we first present an example of the eigenimages using the adjusted spectrum images of dataset D1 shown in Table 3. In this example, 10 and 50 are set for the training size of each class and the reduced dimension, i.e., $d$ in Equation (8), respectively. The example of the eigenimages of dataset D1 is demonstrated in Figure 14.

With $\gamma=0.9$ defined in Equation (8) for dimension reduction, datasets D1-D4 are applied for IF severity detection and the results are shown in Table 5; datasets E1-E4 are used to carry out BF severity detection and the results are shown in Table 6; datasets F1-F4 are employed to conduct OF severity detection and the results are shown in Table 7; and datasets G1-G5 are utilized to evaluate the performance of multifault severity detection and the results are shown in Table 8. Every mentioned test routine is performed for 20 times to obtain the average classification accuracy, where training samples are randomly selected.

As demonstrated in Tables 5-8, acceptable classification accuracies could be achieved with the presented method, especially in case of IF and OF severity detection. From Tables 5 and 7, we can see that the classification accuracies achieve $100 \%$ with only 5 training samples per class. As illustrated in Table 6, the classification accuracy is still over $90 \%$ in most tests, but around $75 \%$ in individual cases. In the experiments with dataset G1-G5, the proposed method also shows its robust capability in handling multiple classification problems.

5.3. Effect of Different Spectrum Resolution for Fault Classification. As presented above, the proposed method exhibits a desirable performance in bearing fault diagnosis. However, there is still one thing to be noted that the spectrum resolution may influence its robustness for applications. Therefore, apart from the selections of CF and image size, it is worthwhile to investigate what frequency resolution is required to generate the original spectrum by specifying an appropriate sampling length as well as an appropriate sampling frequency.

In order to explore the effect of different spectrum resolution for fault classification, the datasets used for determining 


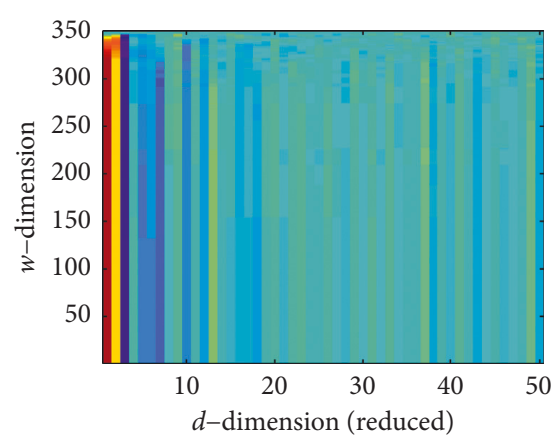

(a)

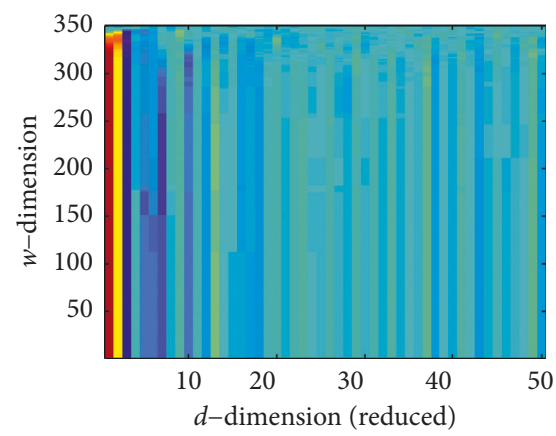

(c)

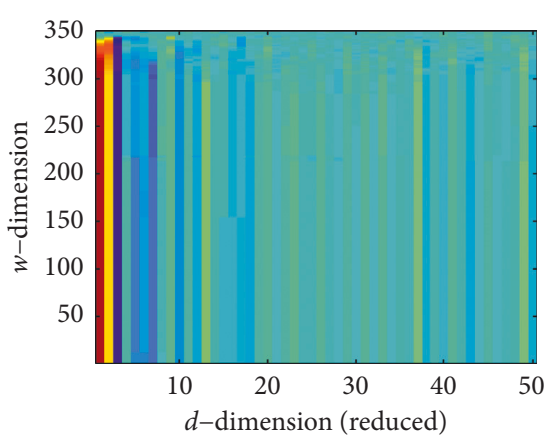

(b)

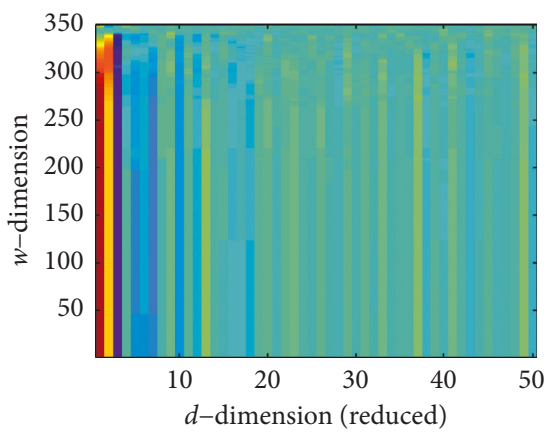

(d)

Figure 10: An example of the eigenimages of dataset A: (a) NO; (b) IF; (c) OF; (d) BF.

TABle 2: Classification results of bearing datasets.

\begin{tabular}{lcc}
\hline Dataset & $\begin{array}{c}\text { The number of } \\
\text { training samples/class }\end{array}$ & Classification accuracy (\%) \\
\hline $\mathrm{A}$ & $1 / 3 / 5 / 10 / 20$ & $94.80 / 97.94 / 99.51 / 99.82 / 100$ \\
$\mathrm{~B}$ & $1 / 3 / 5 / 10 / 20$ & $100 / 100 / 100 / 100 / 100$ \\
$\mathrm{C}$ & $1 / 3 / 5 / 10 / 20$ & $99.95 / 100 / 100 / 100 / 100$ \\
\hline
\end{tabular}

the parameters of $\mathrm{CF}$ and image size, i.e., the dataset $\mathrm{A}$ of experiment I and the dataset G5 of experiment II are also employed to make a further investigation. In this research, considered the computational efficiency of FFT algorithm with Matlab software, different signal lengths of power of two, i.e., $2^{m}$ ( $m$ is a positive integer), are applied to calculate the original spectrum via $N$-point DFT algorithm (here $N=2^{m}$ ). Thus, the frequency resolution of the original spectrum can be expressed as $\Delta f=F_{s} / 2^{m}$. Herein, the value of $m \in$ $\{5,6,7,8,9,10,11,12,13,14\}$ is considered in increasing order.

For dataset $\mathrm{A}$ of experiment $\mathrm{I}$ (sampling rate: $F_{\mathrm{s}}=$ $20000 \mathrm{~Hz}$ ), the frequency resolution series is

$$
\begin{aligned}
\Delta f \in & \{625.00,312.50,156.25,78.13,39.06,19.53,9.77, \\
& 4.88,2.44,1.22\} \mathrm{Hz} .
\end{aligned}
$$

For dataset G5 of experiment II (sampling rate: $F_{\mathrm{s}}=12000 \mathrm{~Hz}$ ), the frequency resolution series is

$$
\begin{aligned}
\Delta f \in & \{375.00,187.50,93.75,46.88,23.44,11.72,5.86, \\
& 2.93,1.46,0.73\} \mathrm{Hz} .
\end{aligned}
$$

With the above spectrum resolution series, the classification performance is tested on dataset A and dataset G5 separately. In this investigation, the performance tests are evaluated with different training size of each health state under each operating condition (i.e., $n_{\text {train }}$ ) as before. That is, $n_{\text {train }}=\{1,3,5,10,20\}$ for dataset $\mathrm{A}$ and $n_{\text {train }}=\{1,3,5,10\}$ for dataset G5. Figures 15 and 16 demonstrate the corresponding classification results in detailed.

Examining the classification accuracies in Figures 15 and 16 , it is noticed that when the training size $n_{\text {train }} \geq 3$, a desirable classification rate can be reached at the spectrum resolution of $19.53 \mathrm{~Hz}$ for experiment I and at the spectrum resolution of $23.44 \mathrm{~Hz}$ for experiment II, respectively. Therefore, we designate the corresponding thresholds of $\Delta f$ for the two experiments, i.e., $\Delta f_{\text {th } 1}=19.6 \mathrm{~Hz}$ for experiment I and $\Delta f_{\text {th2 }}=23.5 \mathrm{~Hz}$ for experiment II. In other words, when the frequency resolution satisfies that $\Delta f<\Delta f_{\text {th1 }}$ for experiment I and $\Delta f<\Delta f_{\text {th2 }}$ for experiment II, the proposed methodology could deliver satisfactory performances, respectively. Hence, a recommendation of the selection of frequency resolution for applying this approach is given as

$$
\Delta f<\min \left\{\Delta f_{\text {thl } 1}, \Delta f_{\text {th } 2}\right\}=19.6 \mathrm{~Hz} .
$$

To further take the computational efficiency of FFT algorithm into consideration, we also recommend that the frequency resolution is not smaller than $1 \mathrm{~Hz}$. As a result, the selection method of spectrum frequency resolution can be determined as

$$
1 \mathrm{~Hz} \leq \Delta f<19.6 \mathrm{~Hz}
$$


TABLE 3: Description of bearing datasets for single-fault severity detection.

\begin{tabular}{|c|c|c|c|c|c|}
\hline Dataset & Training data & Testing data & The number of samples & Fault severity* & Classification label \\
\hline \multirow{4}{*}{$\begin{array}{l}\mathrm{D} 1 / \mathrm{D} 2 / \\
\mathrm{D} 3 / \mathrm{D} 4\end{array}$} & \multirow{4}{*}{$\begin{array}{c}\text { Load 0/Load 1/ } \\
\text { Load 2/Load } 3\end{array}$} & \multirow{4}{*}{ Load $0-3$} & $50 / 50 / 50 / 50$ & Normal & 1 \\
\hline & & & $50 / 50 / 50 / 50$ & 0.007 in & 2 \\
\hline & & & $50 / 50 / 50 / 50$ & 0.014 in & 3 \\
\hline & & & $50 / 50 / 50 / 50$ & 0.021 in & 4 \\
\hline \multirow{4}{*}{$\begin{array}{l}\mathrm{E} 1 / \mathrm{E} 2 / \\
\mathrm{E} 3 / \mathrm{E} 4\end{array}$} & \multirow{4}{*}{$\begin{array}{l}\text { Load } 0 / \text { Load } 1 / \\
\text { Load } 2 / \text { Load } 3\end{array}$} & \multirow{4}{*}{ Load 0-3 } & $50 / 50 / 50 / 50$ & Normal & 1 \\
\hline & & & $50 / 50 / 50 / 50$ & 0.007 ball & 2 \\
\hline & & & $50 / 50 / 50 / 50$ & 0.014 ball & 3 \\
\hline & & & $50 / 50 / 50 / 50$ & 0.021 ball & 4 \\
\hline \multirow{4}{*}{$\begin{array}{l}\text { F1/F2/ } \\
\text { F3/F4 }\end{array}$} & \multirow{4}{*}{$\begin{array}{l}\text { Load 0/Load 1/ } \\
\text { Load 2/Load } 3\end{array}$} & \multirow{4}{*}{ Load $0-3$} & $50 / 50 / 50 / 50$ & Normal & 1 \\
\hline & & & $50 / 50 / 50 / 50$ & 0.007 out & 2 \\
\hline & & & $50 / 50 / 50 / 50$ & 0.014 out & 3 \\
\hline & & & $50 / 50 / 50 / 50$ & 0.021 out & 4 \\
\hline
\end{tabular}

*Among the labels of fault severities, the so-called normal means the testing bearing is normal. 0.007 in means inner-race fault diameter of 0.007 inches; 0.007 ball means ball fault diameter of 0.007 inches; and 0.007 out means outer-race fault diameter of 0.007 inches, etc.

TABLE 4: Description of bearing datasets for multifault severity detection.

\begin{tabular}{|c|c|c|c|c|c|}
\hline Dataset & Training data & Testing data & The number of samples & Fault type and severity & Classification label \\
\hline \multirow{10}{*}{ G1/G2/G3/G4/G5 } & \multirow{10}{*}{$\begin{array}{l}\text { Load 0/Load 1/Load 2/ } \\
\text { Load 3/Load 0-3 }\end{array}$} & \multirow{10}{*}{ Load $0-3$} & $50 / 50 / 50 / 50 / 200$ & Normal & 1 \\
\hline & & & $50 / 50 / 50 / 50 / 200$ & 0.007 in & 2 \\
\hline & & & $50 / 50 / 50 / 50 / 200$ & 0.014 in & 3 \\
\hline & & & $50 / 50 / 50 / 50 / 200$ & 0.021 in & 4 \\
\hline & & & $50 / 50 / 50 / 50 / 200$ & 0.007 ball & 5 \\
\hline & & & $50 / 50 / 50 / 50 / 200$ & 0.014 ball & 6 \\
\hline & & & $50 / 50 / 50 / 50 / 200$ & 0.021 ball & 7 \\
\hline & & & $50 / 50 / 50 / 50 / 200$ & 0.007 out & 8 \\
\hline & & & $50 / 50 / 50 / 50 / 200$ & 0.014 out & 9 \\
\hline & & & $50 / 50 / 50 / 50 / 200$ & 0.021 out & 10 \\
\hline
\end{tabular}

Normal means the testing bearing is normal. 0.007 in means inner-race fault diameter of 0.007 inches; 0.007 ball means ball fault diameter of 0.007 inches; and 0.007 out means outer-race fault diameter of 0.007 inches, etc.

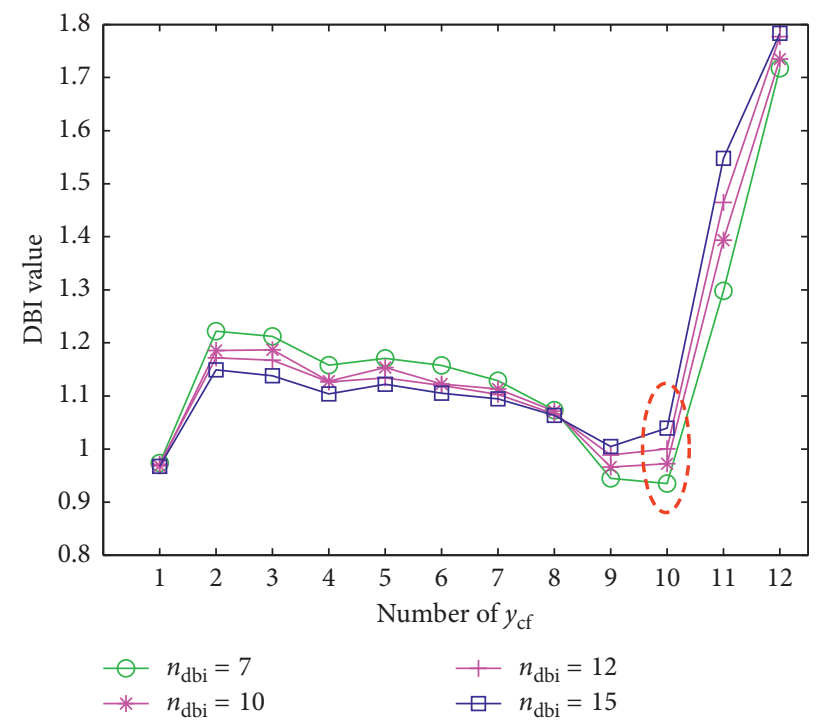

FIGURE 11: Experiment II: the DBI values for $R_{x}=\left[\begin{array}{ll}0 & 210\end{array}\right]$ and $R_{y}=\left[\begin{array}{ll}0 & y_{\mathrm{cf}}\end{array}\right]$.

As for the validation experiments presented in Sections 5.1 and 5.2, the frequency resolutions are $\Delta f=20000 /$ $1024=19.53 \mathrm{~Hz}$ in experiment $\mathrm{I}$ and $\Delta f=12000 / 1024=$ $11.72 \mathrm{~Hz}$ in experiment II, which satisfy the requirement of limiting condition on spectrum resolution.
5.4. Comparisons of Classification Performance on CWRU Datasets. In order to evaluate the performance of the proposed method using the adjusted spectrum images for bearing diagnosis, a comparative study is carried out between current work and some recent publications, 


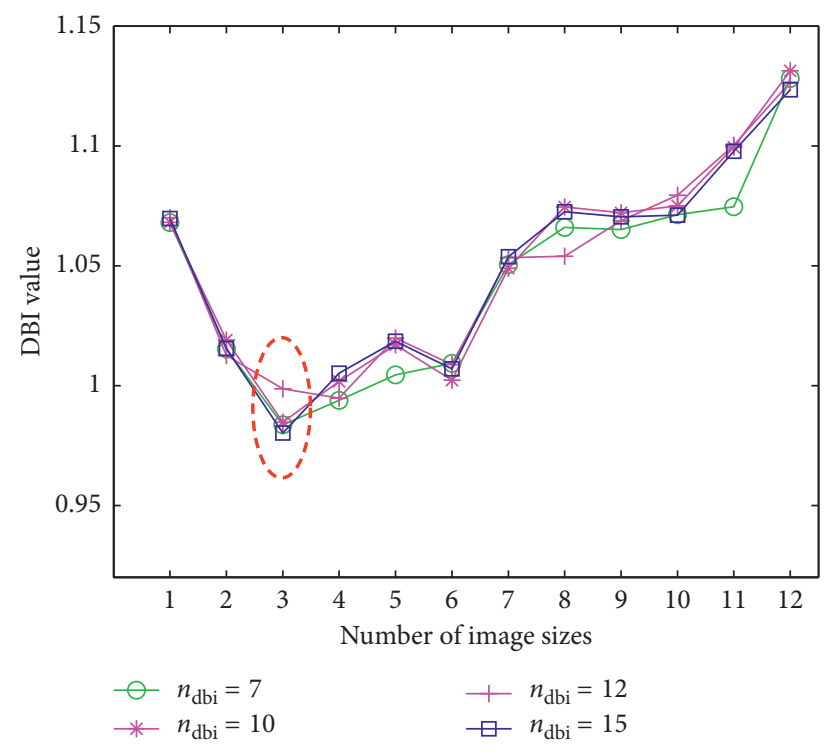

FIgURE 12: Experiment II: the DBI values for different image sizes.

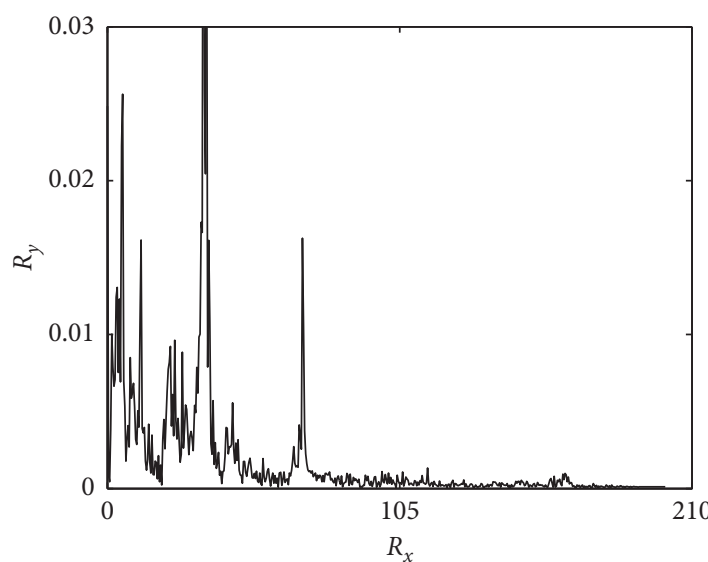

(a)

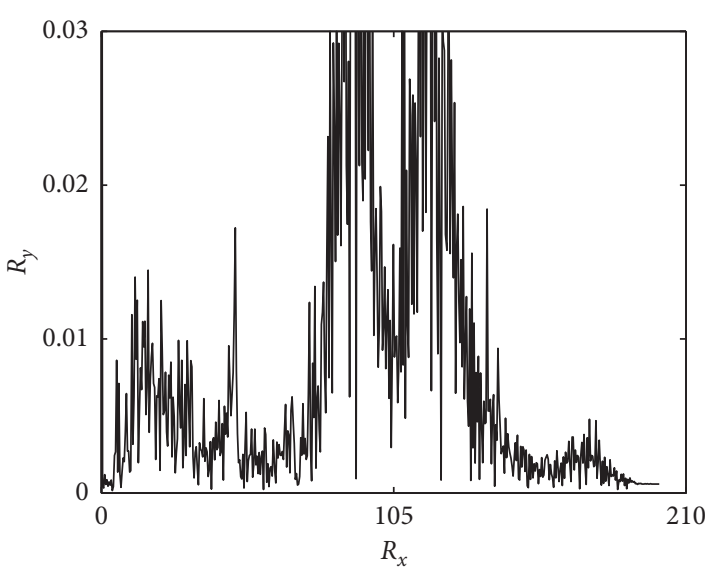

(c)

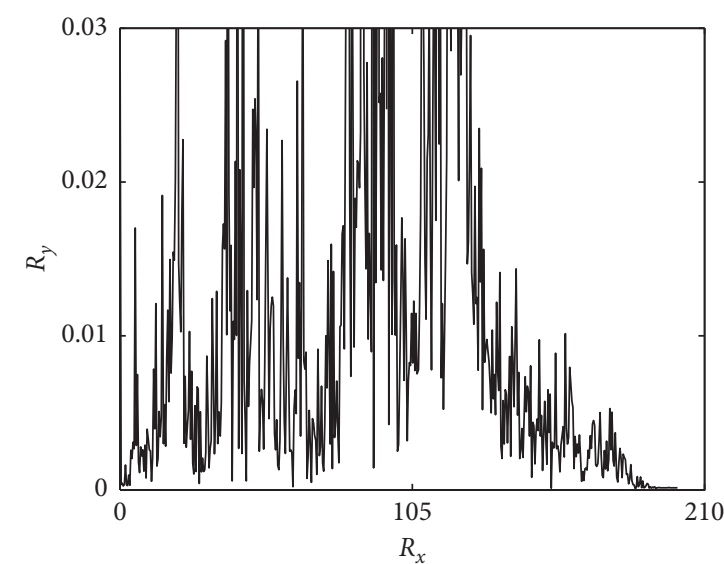

(b)

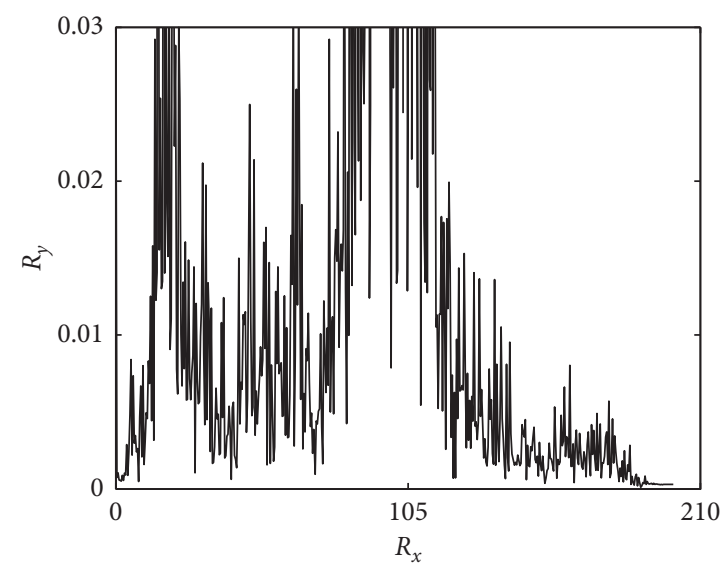

(d)

FIGURE 13: The adjusted spectrum images of D1 with the optimized CF: (a) normal; (b) 0.007 in; (c) 0.014 in; (d) 0.021 in. 


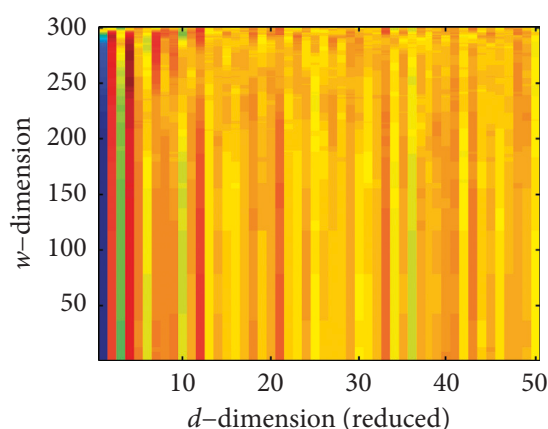

(a)

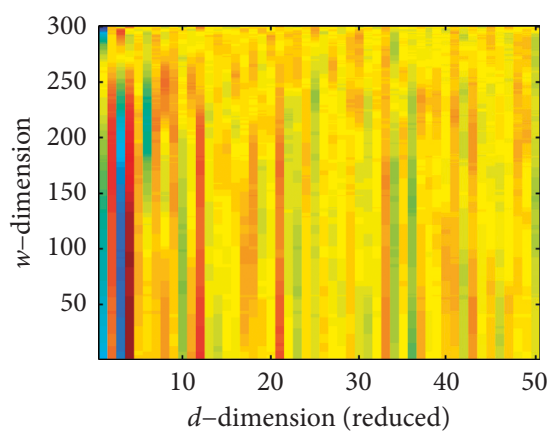

(c)

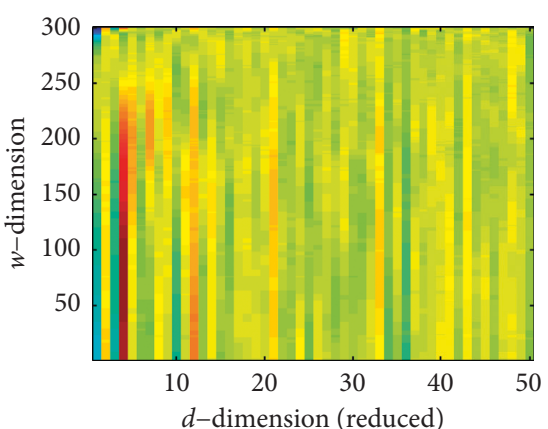

(b)

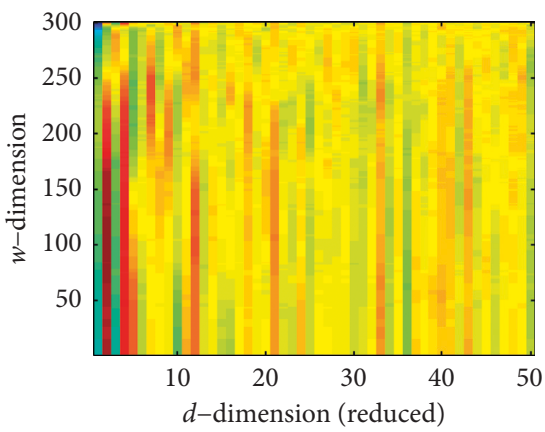

(d)

Figure 14: An example of the eigenimages of dataset D1: (a) normal; (b) 0.007 in; (c) 0.014 in; (d) 0.021 in.

TABLE 5: The classification accuracy for inner-race fault severity detection.

\begin{tabular}{lccccc}
\hline \multirow{2}{*}{ Dataset } & $n^{*}$ & \multicolumn{4}{c}{ Testing accuracy (\%) } \\
& & Load 0 & Load 1 & Load 2 & Load 3 \\
\hline \multirow{3}{*}{ D1 } & 1 & 100 & 100 & 100 & 99.65 \\
& 3 & 100 & 100 & 100 & 99.60 \\
& 5 & 100 & 100 & 100 & 99.85 \\
& 10 & 100 & 100 & 100 & 100 \\
\hline \multirow{4}{*}{ D2 } & 1 & 99.92 & 100 & 100 & 100 \\
& 3 & 99.97 & 100 & 100 & 100 \\
& 5 & 100 & 100 & 100 & 100 \\
& 10 & 100 & 100 & 100 & 100 \\
\hline \multirow{5}{*}{ D3 } & 1 & 98.42 & 100 & 100 & 100 \\
& 3 & 99.28 & 100 & 100 & 100 \\
& 5 & 99.45 & 100 & 100 & 100 \\
& 10 & 100 & 100 & 100 & 100 \\
\hline \multirow{2}{*}{ D4 } & 1 & 99.78 & 100 & 100 & 100 \\
& 3 & 99.88 & 100 & 100 & 100 \\
& 5 & 100 & 100 & 100 & 100 \\
& 10 & 100 & 100 & 100 & 100 \\
\hline
\end{tabular}

${ }^{*} n$ is the training samples per class under each of the specified load conditions.

where the authors adopted the same CWRU bearing datasets.

In $[16,17]$, different methods for extracting features and detecting single-fault severity of bearings are proposed and tested with the datasets in Table 3. In [16], manifold regularization-based semisupervised learning (SSL) is introduced into fault diagnosis of bearings. In [17], bearing diagnosis is performed by applying SVMs and fractal
TABLE 6: The classification accuracy for ball fault severity detection.

\begin{tabular}{lccccc}
\hline \multirow{2}{*}{ Dataset } & $n$ & \multicolumn{4}{c}{ Testing accuracy (\%) } \\
& & Load 0 & Load 1 & Load 2 & Load 3 \\
\hline \multirow{3}{*}{ E1 } & 1 & 97.83 & 95.50 & 97.72 & 89.92 \\
& 3 & 100 & 99.28 & 98.65 & 96.58 \\
& 5 & 100 & 99.63 & 99.38 & 97.47 \\
& 10 & 100 & 100 & 99.58 & 98.20 \\
\hline \multirow{4}{*}{ E2 } & 1 & 99.65 & 100 & 99.97 & 99.95 \\
& 3 & 100 & 100 & 100 & 100 \\
& 5 & 100 & 100 & 100 & 100 \\
& 10 & 100 & 100 & 100 & 100 \\
\hline & 1 & 69.40 & 99.15 & 100 & 100 \\
E3 & 3 & 74.25 & 99.70 & 100 & 100 \\
& 5 & 76.50 & 99.78 & 100 & 100 \\
& 10 & 76.83 & 100 & 100 & 100 \\
\hline \multirow{4}{*}{ E4 } & 1 & 76.83 & 98.58 & 100 & 100 \\
& 3 & 74.40 & 98.88 & 100 & 100 \\
& 5 & 74.80 & 98.25 & 100 & 100 \\
& 10 & 74.97 & 97.33 & 100 & 100 \\
\hline
\end{tabular}

$n$ is the training samples per class under each of the specified load conditions.

dimension. The comparisons are presented in Table 9. The proposed method achieves similar classification accuracy with the results in $[16,17]$ for IF and OF severity detection, but better performance is obtained for BF severity detection with the proposed method.

Similar comparisons for multifault severity detection are also carried out based on the results in some published literatures. DNN is utilized to diagnose the faults of bearings in [9], which deeply exploits the characteristics of vibration 
TABLE 7: The classification accuracy for outer-race fault severity detection.

\begin{tabular}{lccccc}
\hline \multirow{2}{*}{ Dataset } & $n$ & \multicolumn{4}{c}{ Testing accuracy (\%) } \\
& & Load 0 & Load 1 & Load 2 & Load 3 \\
\hline \multirow{3}{*}{ F1 } & 1 & 100 & 100 & 100 & 100 \\
& 3 & 100 & 100 & 100 & 100 \\
& 5 & 100 & 100 & 100 & 100 \\
& 10 & 100 & 100 & 100 & 100 \\
\hline & 1 & 100 & 100 & 100 & 100 \\
F2 & 3 & 100 & 100 & 100 & 100 \\
& 5 & 100 & 100 & 100 & 100 \\
& 10 & 100 & 100 & 100 & 100 \\
\hline & 1 & 100 & 100 & 100 & 100 \\
F3 & 3 & 100 & 100 & 100 & 100 \\
& 5 & 100 & 100 & 100 & 100 \\
& 10 & 100 & 100 & 100 & 100 \\
\hline \multirow{3}{*}{ F4 } & 1 & 100 & 100 & 100 & 100 \\
& 3 & 100 & 100 & 100 & 100 \\
& 5 & 100 & 100 & 100 & 100 \\
& 10 & 100 & 100 & 100 & 100 \\
\hline
\end{tabular}

$n$ is the training samples per class under each of the specified load conditions.

TABLE 8: The classification accuracy for multiclass fault severity detection.

\begin{tabular}{|c|c|c|c|c|c|}
\hline \multirow{2}{*}{ Dataset } & \multirow{2}{*}{$n$} & \multicolumn{4}{|c|}{ Testing accuracy (\%) } \\
\hline & & Load 0 & Load 1 & Load 2 & Load 3 \\
\hline \multirow{4}{*}{ G1 } & 1 & 98.54 & 93.97 & 91.17 & 89.48 \\
\hline & 3 & 99.87 & 98.04 & 96.58 & 94.33 \\
\hline & 5 & 100 & 98.79 & 97.81 & 95.56 \\
\hline & 10 & 100 & 99.24 & 99.48 & 97.98 \\
\hline \multirow{4}{*}{ G2 } & 1 & 98.44 & 100 & 99.92 & 99.92 \\
\hline & 3 & 99.61 & 100 & 99.97 & 100 \\
\hline & 5 & 99.63 & 100 & 100 & 100 \\
\hline & 10 & 99.98 & 100 & 100 & 100 \\
\hline \multirow{4}{*}{ G3 } & 1 & 80.48 & 99.69 & 99.96 & 100 \\
\hline & 3 & 86.11 & 99.78 & 100 & 100 \\
\hline & 5 & 88.97 & 99.89 & 100 & 100 \\
\hline & 10 & 92.72 & 99.95 & 100 & 100 \\
\hline \multirow{4}{*}{ G4 } & 1 & 84.21 & 98.14 & 99.76 & 100 \\
\hline & 3 & 83.24 & 99.00 & 100 & 100 \\
\hline & 5 & 84.19 & 99.45 & 100 & 100 \\
\hline & 10 & 84.08 & 99.70 & 100 & 100 \\
\hline \multirow{4}{*}{ G5 } & 1 & 99.56 & 100 & 99.98 & 100 \\
\hline & 3 & 100 & 100 & 100 & 100 \\
\hline & 5 & 100 & 100 & 100 & 100 \\
\hline & 10 & 100 & 100 & 100 & 100 \\
\hline
\end{tabular}

$n$ is the training samples per class under each of the specified load conditions.

signals. In [18], reconstructed phase space (RPS) and Gaussian mixture model (GMM) are applied for fault diagnosis of bearings. In [19], lifting wavelet packet transform (LWPT) and binary tree system are employed to realize bearing fault diagnosis. In [20], the authors utilized multiscale feature extraction (MFE) and multiclass support vector machine (MSVM) for bearing fault feature extraction and classification, respectively. In [21], trace ratio criterion LDA (TR-LDA) and kNN classifier are used for

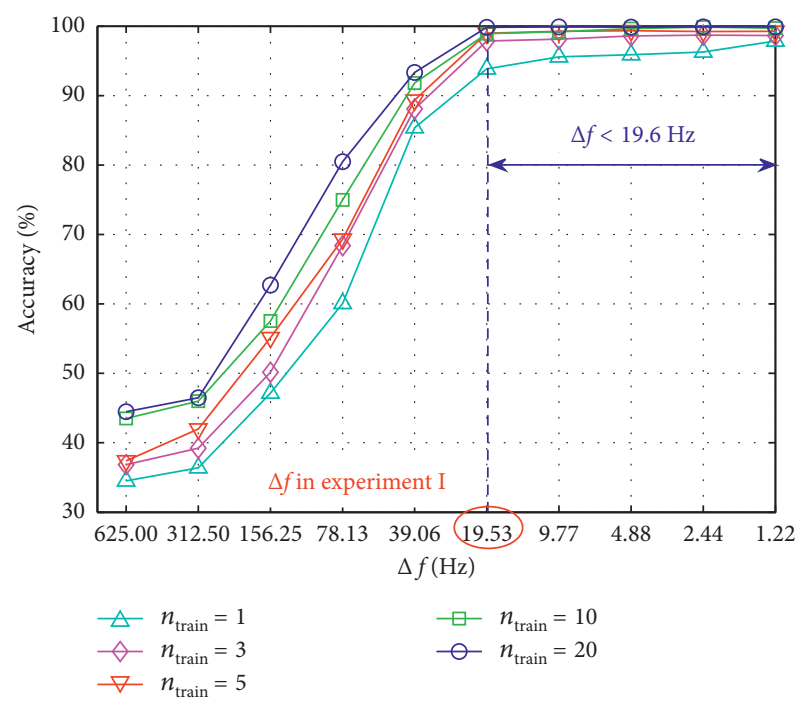

FIgURE 15: Classification performance of dataset A in experiment I with different spectrum resolution.

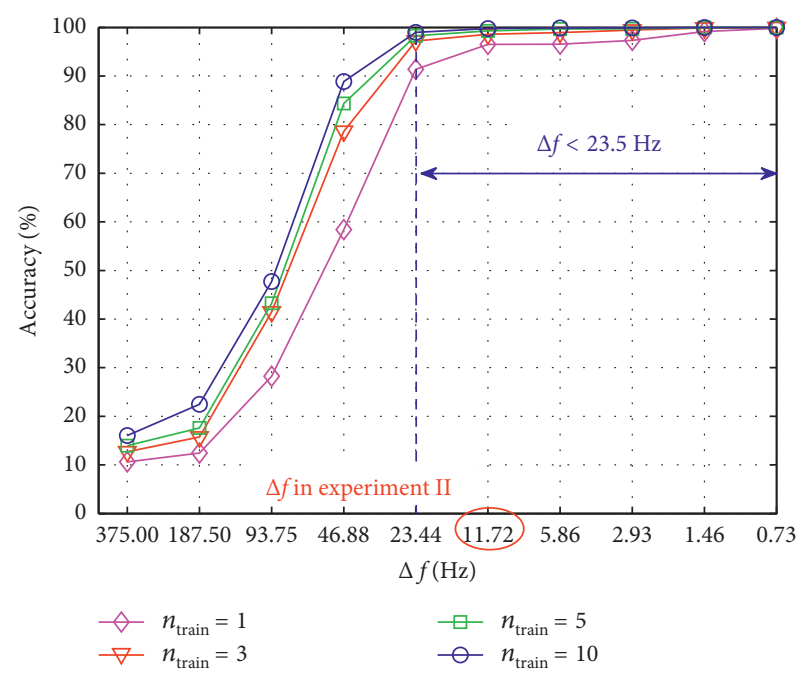

FIGURE 16: Classification performance of dataset G5 in experiment II with different spectrum resolution.

feature reduction and fault classification to realize multifault severity detection of bearings. In [22], the authors applied multiple adaptive neurofuzzy inference system (ANFIS) and genetic algorithm (GA) for the fault diagnosis of bearings. Also in [23], modifed kernel marginal Fisher analysis (MKMFA) is used for feature extraction, and kNN is applied to classify the bearing fault pattern. As shown in Table 10, the classification accuracy of the proposed method could achieve $100 \%$ with 10 training samples per class under the same operating conditions, where its classification results are even not inferior to that obtained with DNN.

In addition, aiming at the complicated ten-class classification problem, nonadjusted spectrum images are also utilized to make a comparison, so as to illustrate the effectiveness of spectrum adjusting. In these tests with nonadjusted spectrum images, the CF for capturing nonadjusted spectra is set to $R_{x}=[06000]$ and $R_{y}=\left[\begin{array}{ll}0 & 0.03\end{array}\right]$ and $\gamma=0.9$ defined in 
TABLE 9: Comparisons for single-fault severity detection between current work and some published work.

\begin{tabular}{|c|c|c|c|c|}
\hline Objective & Method in refs. & Training data & Testing data & Classification accuracy* (\%) \\
\hline \multirow{10}{*}{$\begin{array}{l}\text { IF severity } \\
\text { detection }\end{array}$} & \multirow{4}{*}{ SSL in [16] } & Load $0(40)$ & Load 0-3 (1560) & 100 \\
\hline & & Load $1(40)$ & Load 0-3 (1560) & 100 \\
\hline & & Load $2(40)$ & Load 0-3 (1560) & 100 \\
\hline & & Load $3(40)$ & Load 0-3 (1560) & 100 \\
\hline & Dataset D1 in present work & Load $0(40)$ & Load 0-3 (760) & 100 \\
\hline & Dataset D2 in present work & Load $1(20)$ & Load $0-3(780)$ & 100 \\
\hline & Dataset D3 in present work & Load $2(40)$ & Load $0-3(760)$ & 100 \\
\hline & Dataset D4 in present work & Load $3(20)$ & Load $0-3(780)$ & 100 \\
\hline & SVMs and fractal dimension in [17] & Load $3(472)$ & Load 3 (472) & 100 \\
\hline & Dataset D4 in present work & Load 3 (12) & Load 3 (188) & 100 \\
\hline \multirow{9}{*}{$\begin{array}{l}\text { BF severity } \\
\text { detection }\end{array}$} & \multirow{4}{*}{ SSL in $[16]$} & Load $0(40)$ & Load 0-3 (1560) & 85.26 \\
\hline & & Load 1 (40) & Load 0-3 (1560) & 96.19 \\
\hline & & Load $2(40)$ & Load 0-3 (1560) & 96.59 \\
\hline & & Load $3(40)$ & Load 0-3 (1560) & 79.49 \\
\hline & SVMs and fractal dimension in [17] & Load 3 (472) & Load 3 (472) & 96.31 \\
\hline & Dataset E1 in present work & Load $0(40)$ & Load $0-3(760)$ & 99.42 \\
\hline & Dataset E2 in present work & Load 1 (12) & Load $0-3(788)$ & 100 \\
\hline & Dataset E3 in present work & Load $2(20)$ & Load $0-3(780)$ & 91.49 \\
\hline & Dataset E4 in present work & Load $3(20)$ & Load $0-3(780)$ & 90.53 \\
\hline \multirow{10}{*}{$\begin{array}{l}\text { OF severity } \\
\text { detection }\end{array}$} & \multirow{4}{*}{ SSL in $[16]$} & Load $0(40)$ & Load 0-3 (1560) & 100 \\
\hline & & Load $1(40)$ & Load 0-3 (1560) & 100 \\
\hline & & Load $2(40)$ & Load 0-3 (1560) & 100 \\
\hline & & Load $3(40)$ & Load 0-3 (1560) & 100 \\
\hline & SVMs and fractal dimension in [17] & Load 3 (472) & Load 3 (472) & 99.89 \\
\hline & Dataset F1 in present work & Load 0 (12) & Load $0-3(788)$ & 100 \\
\hline & Dataset F2 in present work & Load 1 (12) & Load $0-3(788)$ & 100 \\
\hline & Dataset F3 in present work & Load 2 (12) & Load $0-3(788)$ & 100 \\
\hline & Dataset F4 in present work & Load 3 (12) & Load $0-3(788)$ & 100 \\
\hline & Dataset F4 in present work & Load 3 (12) & Load 3 (188) & 100 \\
\hline
\end{tabular}

${ }^{*}$ The classification accuracy is determined by no. of successfully classified samples/No. of total testing samples $\times 100 \%$ under specified load condition.

TABle 10: Comparisons for multiclass fault detection between current work and some published work.

\begin{tabular}{|c|c|c|c|}
\hline Method in refs. & Training data & Testing data & Classification accuracy* (\%) \\
\hline \multirow[t]{2}{*}{ RPS and GMM in [18] } & Load $1(3840)$ & Load $1(3840)$ & $99.95^{\mathrm{a}}$ \\
\hline & Load $1(1000)$ & Load $1(1000)$ & 99.95 \\
\hline \multirow{3}{*}{ DNN-based method in [9] } & Load 2 (1000) & Load $2(1000)$ & 99.61 \\
\hline & Load 3 (1000) & Load 3 (1000) & 99.74 \\
\hline & Load $1-3(3000)$ & Load $1-3(3000)$ & 99.68 \\
\hline LWPT and binary tree system in [19] & Load $0(400)$ & Load $0(200)$ & 99.53 \\
\hline MFE and MSVM in [20] & Load $0(840)$ & Load $0(360)$ & 94.50 \\
\hline \multirow[t]{2}{*}{ TR-LDA2 and kNN classifier in [21] } & Load $1(200)$ & Load $1(600)$ & 98.00 \\
\hline & Load $2(150)$ & Load $2(650)$ & 97.65 \\
\hline Multiple ANFIS combination in [22] & Load $0-3(300)$ & Load $0-3(300)$ & 91.33 \\
\hline MKMFA and kNN in [23] & Load $0-3(500)$ & Load $0-3(500)$ & 97.45 \\
\hline Dataset G2 in present work & Load $1(100)$ & Load $1(400)$ & 100 \\
\hline Dataset G3 in present work & Load $2(100)$ & Load $2(400)$ & 100 \\
\hline Dataset G4 in present work & Load $3(100)$ & Load $3(400)$ & 100 \\
\hline Dataset G1 in present work & Load $0(100)$ & Load 0-3 (1900) & 99.13 \\
\hline Dataset G2 in present work & Load $1(100)$ & Load 0-3 (1900) & 99.99 \\
\hline Dataset G3 in present work & Load $2(100)$ & Load 0-3 (1900) & 98.07 \\
\hline Dataset G4 in present work & Load $3(100)$ & Load 0-3 (1900) & 95.72 \\
\hline Dataset G5 in present work & Load $0-3(400)$ & Load 0-3 (1600) & 100 \\
\hline Dataset G1 with nonadjusted spectrum ${ }^{\mathrm{b}}$ & Load $0(100)$ & Load $0-3(1900)$ & 84.97 \\
\hline Dataset G2 with nonadjusted spectrum ${ }^{\mathrm{b}}$ & Load $1(100)$ & Load $0-3(1900)$ & 92.30 \\
\hline Dataset G3 with nonadjusted spectrum ${ }^{\mathrm{b}}$ & Load $2(100)$ & Load $0-3(1900)$ & 89.66 \\
\hline Dataset G4 with nonadjusted spectrum ${ }^{\mathrm{b}}$ & Load $3(100)$ & Load 0-3 (1900) & 90.72 \\
\hline Dataset G5 with nonadjusted spectrum ${ }^{\mathrm{b}}$ & Load $0-3(400)$ & Load $0-3(1600)$ & 100 \\
\hline
\end{tabular}

a This classification accuracy is computed based on Table 2 in [18], which is the average of the ten classes. ${ }^{\text {b}}$ The test settings are same with that in G1-G5 using the adjusted spectrum images. 
(8). Each experiment (10 samples/class/load for training) is repeated 20 times to acquire the average accuracy. As depicted in Table 10, for dataset G1-G5, classification rates with $84.97 \%, 92.30 \%, 89.66 \%, 90.72 \%$, and $100 \%$ are achieved, respectively, with nonadjusted spectrum images. But with the proposed method for spectrum adjusting, higher classification rates with $99.13 \%, 99.99 \%, 98.07 \%, 95.72 \%$, and $100 \%$ could be reached for datasets G1-G5, respectively.

It is clear that the proposed spectrum adjusting scheme could suppress the interference of rotating speed and reduce the changes of diagram structure, which facilitates that this approach could obtain excellent classification accuracies under different operating conditions. The robustness of fault diagnosis could also be highlighted using the adjusted image as feature.

\section{Discussion}

The adjusted spectrum image is indeed a $2 \mathrm{D}$ representation of the $1 \mathrm{D}$ vector of FFT coefficients. In [9], the vector of FFT coefficients is utilized as the inputs of DNNs, and the achieved classification accuracy is $99.68 \%$ with a large number of training samples. By using the proposed 2D adjusted spectrum images together with a simple NNC classifier, the classification accuracy is $100 \%$ and the number of required training samples is much smaller (Table 10). From this point of view, such a 2D representation in the form of image contains more useful information for bearing fault diagnosis.

The experimental results also demonstrate that the proposed method could diagnose bearing faults effectively under different operating conditions, even with very limited training samples. The training samples in dataset D, E, F, G1, G2, G3, and G4 are only from one operating condition, while the testing samples are from four different operating conditions (Tables 3 and 4). In these cases, high classification accuracies are still achieved with the proposed method as shown in Tables 5-7. It can also be observed that, a relatively high classification accuracy is also obtained even with one single training sample (Table 8). For the fault pattern classification with limited training samples, artificial intelligence classifiers (e.g., ANN, SVM) may demonstrate worse performance than NNC because there commonly are needed large amounts of samples to obtain the model parameters. Here, taking datasets G1-G4 for example, Table 11 presents their classification performance with NNC and SVM (using polynomial kernel function) under limited training sample condition. According to the comparison results, when there only exist 10 training samples for single-fault type, SVM is inferior to NNC due to lacking of enough training samples to optimize SVM parameters. This is the main reason why NNC is selected as the classifier for fault classification in this work.

It is worth mentioning that the proposed method belongs to the scope of fault classification of bearings; therefore, the training samples must be obtained in advance. However, thanks to the spectrum adjusted by the rotating speed frequency, the geometrical structure of the spectrum image is not significantly influenced by the speed. Therefore, the training data need not contain samples of all operating conditions, but contain those of all fault types. When the
TABLE 11: Performance comparison between NNC and SVM under limited training sample condition.

\begin{tabular}{lcccc}
\hline Dataset & Training data & Testing data & \multicolumn{2}{c}{$\begin{array}{c}\text { Classification } \\
\text { accuracy }\end{array}$} \\
\hline \multirow{2}{*}{ G1 } & Load 0 (100) & Load 0-3 (1900) & NNC & $99.13 \%$ \\
& & & SVM & $95.05 \%$ \\
G2 & Load 1 (100) & Load 0-3 (1900) & NNC & $99.99 \%$ \\
& & & SVM & $95.32 \%$ \\
G3 & Load 2 (100) & Load 0-3 (1900) & NNC & $98.07 \%$ \\
& & & SVM & $94.53 \%$ \\
G4 & Load 3 (100) & Load 0-3 (1900) & NNC & $95.72 \%$ \\
& & & SVM & $90.26 \%$ \\
\hline
\end{tabular}

geometrical structures of bearing spectra are similar for a same failure type, those bearings could be diagnosed with the proposed method. Furthermore, the proposed feature is easier to compute and postprocess compared with References $[6,7]$ because the presented spectrum image is a binary image which avoids performing binary conversion. All these characteristics promote it become a robust feature for bearing fault diagnosis.

\section{Conclusions}

In this paper, the adjusted spectrum image is proposed as feature for bearing fault diagnosis under different operation conditions. First, the validity indicator, DBI, is introduced to optimize the CF and image size for capturing spectrum images. Then with the optimized CF and image-size, the adjusted spectrum images are generated via FFT according to the rotating speed and 2DPCA is applied to reduce the image dimension for facilitating classification with an NNC. Two data examples are employed to verify the effectiveness of the proposed approach. On the base of these bearing data, the effect of spectrum frequency resolution is further investigated and a recommended selection method is presented to determine the frequency resolution. In the proposed method, the training samples could be from one operating condition, while the testing samples could be from different operating conditions. Experimental results illustrate that this methodology could achieve high classification accuracy with very limited training samples.

\section{Data Availability}

Some experimental data used in this study are acquired from the bearing data centre of CWRU and the web page http://csegroups.case.edu/bearingdatacenter/home (accessed September 2018). The other data used to support the fndings are available from the corresponding author upon request.

\section{Conflicts of Interest}

The authors declare that they have no conflicts of interest.

\section{Acknowledgments}

The work was supported by National Key R\&D Program of China (grant no. 2016YFC0802908), National Natural 
Science Foundation of China (grant nos. 51475455 and 51605478), Natural Science Foundation of Jiangsu Province (grant no. BK20160251), and the Project Funded by the Priority Academic Program Development of Jiangsu Higher Education Institutions (PAPD).

\section{References}

[1] R. B. Randall and J. Antoni, "Rolling element bearing diagnostics-a tutorial," Mechanical Systems and Signal Processing, vol. 25, no. 2, pp. 485-520, 2011.

[2] X. Liu, L. Bo, X. He, and M. Veidt, "Application of correlation matching for automatic bearing fault diagnosis," Journal of Sound and Vibration, vol. 331, no. 26, pp. 5838-5852, 2012.

[3] J. Zarei, M. A. Tajeddini, and H. R. Karimi, "Vibration analysis for bearing fault detection and classification using an intelligent filter," Mechatronics, vol. 24, no. 2, pp. 151-157, 2014.

[4] A. Rai and S. Upadhyay, "A review on signal processing techniques utilized in the fault diagnosis of rolling element bearings," Tribology International, vol. 96, pp. 289-306, 2016.

[5] W. Li, M. Qiu, Z. Zhu, B. Wu, and G. Zhou, "Bearing fault diagnosis based on spectrum images of vibration signals," Measurement Science and Technology, vol. 27, no. 3, p. 035005, 2016.

[6] M. Amar, I. Gondal, and C. Wilson, "Vibration spectrum imaging: a novel bearing fault classification approach," IEEE Transactions on Industrial Electronics, vol. 62, no. 1, pp. 494-502, 2015.

[7] R. Klein, E. Masad, E. Rudyk, and I. Winkler, "Bearing diagnostics using image processing methods," Mechanical Systems and Signal Processing, vol. 45, no. 1, pp. 105-113, 2014.

[8] J. Griffaton, J. Picheral, and A. Tenenhaus, "Enhanced visual analysis of aircraft engines based on spectrograms," in Proceedings of International Conference on Noise and Vibration Engineering (ISMA2014) and International Conference on Uncertainty in Structural Dynamics (USD2014), pp. 27952808, Leuven, Belgium, September 2014.

[9] F. Jia, Y. Lei, J. Lin, X. Zhou, and N. Lu, "Deep neural networks: a promising tool for fault characteristic mining and intelligent diagnosis of rotating machinery with massive data," Mechanical Systems and Signal Processing, vol. 72-73, pp. 303-315, 2016.

[10] W. Lu, X. Wang, C. Yang, and T. Zhang, "A novel feature extraction method using deep neural network for rolling bearing fault diagnosis," in Proceedings of 27th Chinese Control and Decision Conference (CCDC), pp. 2427-2431, Qingdao, China, May 2015.

[11] H. Shao, H. Jiang, X. Zhang, and M. Niu, "Rolling bearing fault diagnosis using an optimization deep belief network," Measurement Science and Technology, vol. 26, no. 11, article 115002, 2015.

[12] C. W. R. University, "Bearings vibration dataset," http:// csegroups.case.edu/bearingdatacenter/home.

[13] D. L. Davies and D. W. Bouldin, "A cluster separation measure," IEEE Transactions on Pattern Analysis and Machine Intelligence, vol. 1, no. 2, pp. 224-227, 1979.

[14] J. Yang, D. Zhang, A. F. Frangi, and J.-y. Yang, "Twodimensional PCA: a new approach to appearance-based face representation and recognition," IEEE Transactions on Pattern Analysis and Machine Intelligence, vol. 26, no. 1, pp. 131-137, 2004.

[15] T. M. Cover and P. E. Hart, "Nearest neighbor pattern classification," IEEE Transactions on Information Theory, vol. 13, no. 1, pp. 21-27, 1967.
[16] J. Yuan and X. Liu, "Semi-supervised learning and condition fusion for fault diagnosis," Mechanical Systems and Signal Processing, vol. 38, no. 2, pp. 615-627, 2013.

[17] J. Yang, Y. Zhang, and Y. Zhu, "Intelligent fault diagnosis of rolling element bearing based on SVMs and fractal dimension," Mechanical Systems and Signal Processing, vol. 21, no. 5, pp. 2012-2024, 2007.

[18] G. F. Wang, Y. B. Li, and Z. G. Luo, "Fault classification of rolling bearing based on reconstructed phase space and Gaussian mixture model," Journal of Sound and Vibration, vol. 323, no. 3-5, pp. 1077-1089, 2009.

[19] L. Zhang, L. Zhang, J. Hu, and G. Xiong, "Bearing fault diagnosis using a novel classifier ensemble based on lifting wavelet packet transforms and sample entropy," Shock and Vibration, vol. 2016, Article ID 4805383, 13 pages, 2016.

[20] M. Zhang, Z. Cai, and W. Cheng, "Multiple-fault diagnosis method based on multiscale feature extraction and MSVM_ PPA," Shock and Vibration, vol. 2018, Article ID 6209371, 12 pages, 2018.

[21] M. Zhao, X. Jin, Z. Zhang, and B. Li, "Fault diagnosis of rolling element bearings via discriminative subspace learning: visualization and classification," Expert Systems with Applications, vol. 41, no. 7, pp. 3391-3401, 2014.

[22] Y. Lei, Z. He, Y. Zi, and Q. Hu, "Fault diagnosis of rotating machinery based on multiple ANFIS combination with GAs," Mechanical Systems and Signal Processing, vol. 21, no. 5, pp. 2280-2294, 2007.

[23] L. Jiang and S. Guo, "Modified kernel marginal Fisher analysis for feature extraction and its application to bearing fault diagnosis," Shock and Vibration, vol. 2016, Article ID 1205868, 16 pages, 2016. 


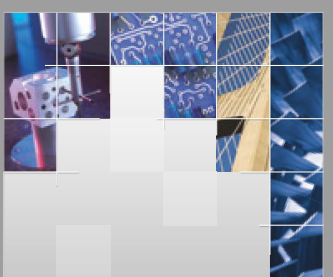

\section{Enfincering}
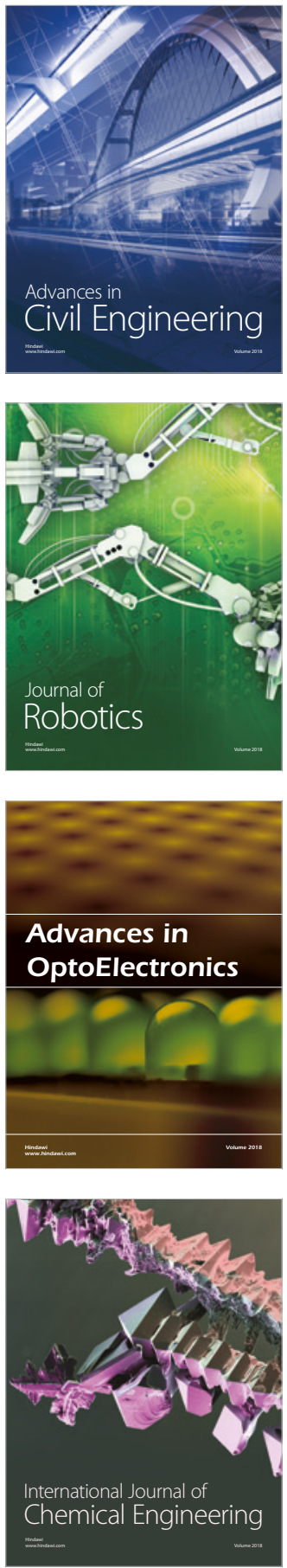

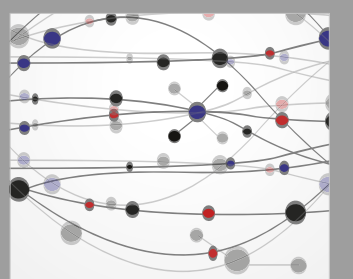

\section{Rotating \\ Machinery}

The Scientific World Journal

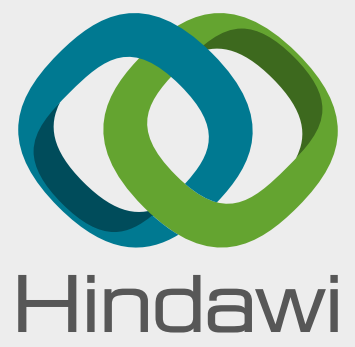

Submit your manuscripts at

www.hindawi.com
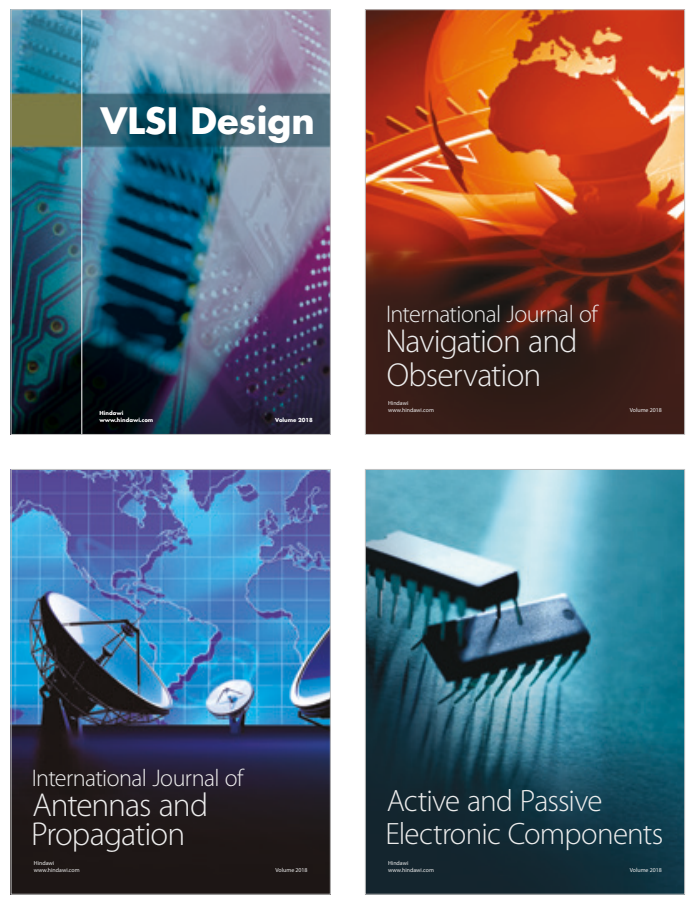
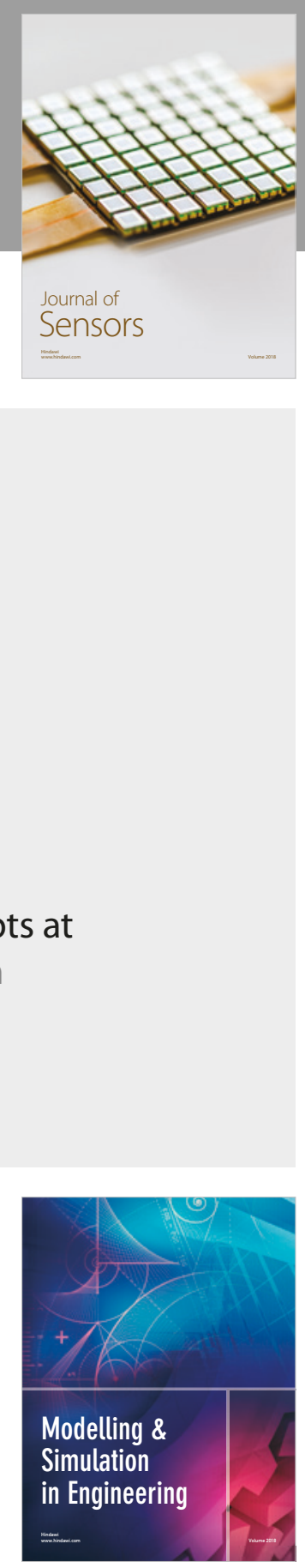

\section{Advances \\ Multimedia}
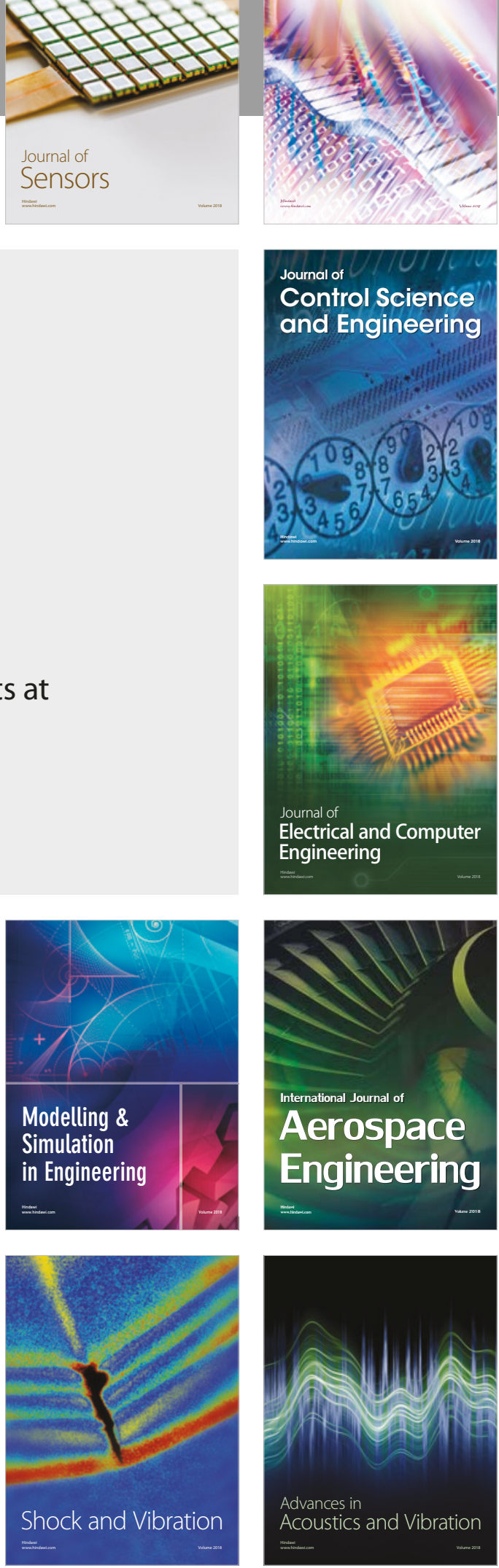